\title{
Corela
}

Cognition, représentation, langage

16-2 | 2018

Vol. $16, n^{\circ} 2$

\section{Défense du complément circonstanciel}

\section{François Trouilleux}

\section{OpenEdition}

\section{Journals}

Electronic version

URL: http://journals.openedition.org/corela/7029

DOI: 10.4000/corela.7029

ISSN: 1638-573X

\section{Publisher}

Cercle linguistique du Centre et de l'Ouest - CerLICO

\section{Electronic reference}

François Trouilleux, «Défense du complément circonstanciel », Corela [Online], 16-2 | 2018, Online since 21 December 2018, connection on 30 April 2019. URL : http://journals.openedition.org/ corela/7029 ; DOI : 10.4000/corela.7029

This text was automatically generated on 30 April 2019.

\section{(c) (i) (2)(2)}

Corela - cognition, représentation, langage est mis à disposition selon les termes de la licence Creative Commons Attribution - Pas d'Utilisation Commerciale - Partage dans les Mêmes Conditions 4.0 International. 


\title{
Défense du complément circonstanciel
}

\author{
François Trouilleux
}

\section{Introduction}

1 Les grands-parents qui aident leurs petits-enfants à faire leurs devoirs de français, souvent, ne retrouvent pas dans ces travaux les concepts qu'on leur avait enseignés dans leur enfance. C'est qu'au cours des dernières décennies, la «nouvelle grammaire » s'est substituée à la "grammaire traditionnelle». On peut dater les débuts du changement, pour ce qui est de l'enseignement en France, des environs de 1973 : cette année-là parait La nouvelle grammaire scolaire, de Dubois et Lagane, et des fascicules d'exercices associés qui affichent en quatrième de couverture : « Pour la première fois, l'enseignement de la grammaire est entièrement conçu à partir de la linguistique moderne » (Marchand et al., 1973).

2 Une des principales innovations de cette nouvelle grammaire est la définition d'une nouvelle ligne de partage entre les compléments que la tradition associait au verbe. Ainsi, pour la grammaire traditionnelle, le complément à l'école est du même type ( «circonstanciel ») dans les phrases Cet enfant va à l'école et Cet enfant travaille bien à l'école, tandis que pour la nouvelle grammaire, ces deux occurrences de à l'école sont des compléments de types différents et il est exclu d'appeler le premier « circonstanciel » (cf. Riegel et al., [1994] 2009, p. 267).

3 Le concept traditionnel de complément circonstanciel est très largement reconnu comme flou par les linguistes. C'est «un domaine spécialement instable de la grammaire » (Leeman, 1998, p. 7). «La notion connait une telle diversité d'emplois qu'elle tend à devenir inutilisable » (Rémi-Giraud, 1998, p. 66). « Les linguistes se querellent autour de la ligne de démarcation [entre compléments d'objet et compléments circonstanciels]. » (Wilmet, 2007, p. 193). Je ne m'intéresserai pas dans cet article à toutes les tentatives de définition du concept ${ }^{1}$, mais plutôt aux définitions qui dominent dans la grammaire traditionnelle et la grammaire scolaire actuelle. Contre la nouvelle nomenclature en 
vogue, mais attaché aux fondamentaux de l'analyse linguistique, je prendrai la défense de la définition traditionnelle, en la nettoyant de ses défauts.

4 La première partie de l'article présente le point de vue de la nouvelle grammaire: un nouveau découpage des données et une nouvelle terminologie ( $\$ 1.1$ ), la définition de la nouvelle répartition par des critères formels d'effacement et déplacement (\$ 1.2), le volet interprétatif, sémantique, auquel la caractérisation syntaxique est censée correspondre (§ 1.3). Après cet exposé, je développerai une critique de ce point de vue en montrant que les définitions syntaxiques et sémantiques ne couvrent pas le même champ d'observation (§ 2.1), qu'on ne peut pas ne pas faire appel au sens et que les critères syntaxiques utilisés ne peuvent entrer de façon pertinente dans le processus de traitement des phrases (\$ 2.2).

5 J'examinerai ensuite la définition traditionnelle du complément circonstanciel à partir de la définition de Grevisse (1980) (§ 3.1) et de l'historique qu'en a fait Chervel $(1977,1979)$ (§ 3.2), puis je relaierai les principales critiques qui en sont faites ( $\$ 3.3$ et 3.4). Le parcours va de Beauzée (1767, t. 2, p. 60-64) aux grammairiens d'aujourd'hui.

6 Je prendrai ensuite la défense du point de vue traditionnel en trois temps. Il n'y a pas lieu de substituer les nouvelles définitions aux anciennes, car elles ne sont pas incompatibles ( $\S$ 4.1). La caractérisation du sens des compléments est rendue nécessaire par l'existence même de compléments non essentiels ( $\$ 4.2$ ). Les définitions originelles du complément circonstanciel avaient une dimension parfaitement compatible avec l'angle d'analyse privilégié qu'est le recours à la commutation en linguistique (\$ 4.3). Enfin, je terminerai en proposant une définition «restaurée» des compléments du verbe : un retour aux sources, avec réparation des imperfections, qui plaide pour une réévaluation de la place accordée à la dimension interprétative dans l'enseignement de la grammaire.

\section{Répartition nouvelle des compléments du verbe}

\subsection{Tradition et modernité : deux découpages de la réalité}

7 A la division traditionnelle des compléments du verbe en compléments d'objet et compléments circonstanciels, les linguistes de la fin $\mathrm{du} \mathrm{xx}^{\mathrm{e}}$ siècle ont substitué une division qu'on peut caractériser comme une opposition entre compléments essentiels et compléments non essentiels, si on considère que tous les compléments en question dépendent du verbe, ou comme une opposition entre compléments du verbe et compléments de phrase, si on considère que la nouvelle répartition est induite par une différence de structure.

Le tableau de la figure 1 met en correspondance de façon schématique les deux répartitions. Je retiens les termes essentiel et non essentiel. D'après Pellat (2009, p. 54), le 
terme complément essentiel a fait son entrée officielle dans la Terminologie grammaticale en 1997.

\begin{tabular}{|l|l|l|}
\hline & \multicolumn{2}{|l|}{ compléments du verbe } \\
\hline découpage traditionnel & compléments d'objet & compléments circonstanciels \\
\hline découpage actuel & compléments essentiels & compléments non essentiels \\
\hline
\end{tabular}

Figure. Deux découpages de la classe des compléments traditionnellement associés au verbe.

Ce tableau est schématique en ce qu'il cible les lignes de partage sur lesquelles la majorité des grammaires anciennes et modernes se focalisent en premier lieu. Il est bien entendu qu'on peut proposer un découpage plus fin - ce que font en général les linguistes; par exemple, Gosselin (1985) distingue parmi les compléments non essentiels les compléments du verbe et les compléments de phrase, distinction qu'on retrouve chez Pellat (2009) sous les noms compléments circonstanciels et compléments de phrase. Il est bien entendu aussi que tous les linguistes ne sont pas d'accord; par exemple, Wilmet (2007) défend un classement en deux CV (compléments du verbe) et cinq CP (compléments de la prédication) avec une ligne de partage entre CV et CP qui est proche du découpage traditionnel. On s'intéressera dans cet article essentiellement aux partitions binaires représentées dans la figure 1, et seulement marginalement aux raffinements à l'intérieur des deux sous-classes principales établies dans chaque tradition.

10 Le point clé du changement est que des compléments circonstanciels (dans le sens traditionnel) sont passés dans la classe des compléments essentiels. C'est le cas de à l'école dans Cet enfant va à l'école. Si ce changement de point de vue est largement partagé, la terminologie l'est moins. La figure 2 donne les termes utilisés dans différentes grammaires pour désigner le complément à l'école dans les deux phrases citées en introduction.

\begin{tabular}{|c|c|c|}
\hline & Cet enfant va à l'école & Cet enfant travaille bien à l'école \\
\hline $\begin{array}{l}\text { Dubois et Lagane } \\
(1973)\end{array}$ & $\begin{array}{l}\text { circonstanciel } \\
\text { complément du groupe du } \\
\text { verbe }\end{array}$ & $\begin{array}{l}\text { circonstanciel } \\
\text { complément de la phrase (p. 153) }\end{array}$ \\
\hline Goosse (1986) & $\begin{array}{l}\text { complément adverbial } \\
\text { essentiel }\end{array}$ & complément adverbial non essentiel \\
\hline Riegel et al. & $\begin{array}{l}\text { complément d'objet } \\
\text { indirect } \\
\text { constituant du groupe } \\
\text { verbal } \\
\text { complément du verbe }\end{array}$ & $\begin{array}{l}\text { circonstanciel (ou circonstant) } \\
\text { constituant de la phrase (p. 243) }\end{array}$ \\
\hline $\begin{array}{l}\text { Chartrand et al. } \\
\text { (1999) }\end{array}$ & $\begin{array}{l}\text { complément indirect } \mathrm{du} \\
\text { verbe }\end{array}$ & complément de $\mathrm{P}$ \\
\hline
\end{tabular}




\begin{tabular}{|l|l|l|}
\hline Pellat (2009) & $\begin{array}{l}\text { complément essentiel du } \\
\text { verbe }\end{array}$ & $\begin{array}{l}\text { complément facultatif du verbe complément } \\
\text { circonstanciel }^{2}\end{array}$ \\
\hline
\end{tabular}

Figure. Différentes désignations de deux compléments.

11 A certains égards, on est en présence d'un chaos terminologique, favorisé par l'invention d'un nouvel emploi du nom complément et par la conservation des anciens termes pour de nouveaux concepts.

Pour ajouter la nouvelle opposition à l'ancienne (qu'ils conservent, sans jamais la définir, comme une donnée acquise), Dubois et Lagane (1973) donnent au nom complément un sens proche de celui de constituant ${ }^{3}$. Dès lors, le mot devient ambigu : dans Cet enfant va à l'école , le groupe à l'école est complément (au sens traditionnel) du verbe et complément (dans le sens de " constituant») du groupe du verbe. Pour la phrase Cet enfant travaille bien à l'école, dire que « à l'école est complément de la phrase " peut alors se comprendre comme (1) à l'école est complément (au sens traditionnel) de la phrase Cet enfant travaille bien (mais alors on a deux objets phrases: Cet enfant travaille bien et Cet enfant travaille bien à l'école) ou (2) que à l'école est constituant de la phrase Cet enfant travaille bien à l'école (c'est ce que veulent dire Dubois et Lagane, mais dans ce cas, de quoi à l'école est-il complément au sens habituel du terme?).

Riegel et al. ([1994] 2009) gardent le terme circonstanciel ou objet direct, mais en leur donnant un nouveau sens, puisqu'ils ne les appliquent pas aux mêmes compléments. Buyssens (1975) ou Monneret (1999) font de même; Pellat (2009) aussi pour le terme circonstanciel. On peut le regretter, car un nouveau découpage des données aurait sans doute mérité une nouvelle terminologie.

Chartrand et al. (1999) sont cohérents, en ce qu'ils rejettent l'ancienne terminologie, mais se trompent quand ils précisent en note (p. 109) que «le complément indirect du verbe est traditionnellement appelé "complément d'objet indirect" » : dans Cet enfant va à l'école, où ils voient un complément indirect du verbe, la tradition n'identifie aucun complément d'objet indirect. Dans le même ordre d'idée, Pellat (2009, p. 55) se trompe aussi lorsqu'il écrit que «la fonction complément circonstanciel de la grammaire scolaire renvoie à deux types de compléments librement ajoutés, riches en informations, mais syntaxiquement facultatifs »: dans Cet enfant va à l'école, pour la grammaire scolaire dont il parle, le complément à l'école était circonstanciel tout en étant obligatoire ${ }^{4}$. A redéfinir des termes, on finit par en oublier ce qu'ils voulaient dire autrefois.

Le plus cohérent parait finalement être Goosse (1986), qui, dans sa révision du Bon Usage de Grevisse ([1936] 1980), adopte la nouvelle ligne de partage via l'opposition essentiel / non essentiel, tout en préservant l'ancienne via le qualificatif adverbial, qui correspond à l'ancien circonstanciel. Certes, il critique l'ancienne terminologie, en précisant que «les compléments adverbiaux essentiels ne méritent certainement pas d'être appelés circonstanciels » (\$301), mais il se garde d'en changer le sens.

Dans cet article, je laisserai à chacun sa terminologie et emploierai pour ma part les termes essentiel et non essentiel pour la nouvelle répartition des compléments du verbe, et le terme complément circonstanciel dans son sens traditionnel. 


\subsection{Définition de l'opposition entre complément essentiels et non essentiels}

17 L'opposition entre compléments essentiels et non essentiels dans la nouvelle grammaire est toujours définie d'abord syntaxiquement, puis sémantiquement.

\subsubsection{Définition sur critères formels}

La linguistique moderne qui a donné naissance à la nouvelle grammaire est constituée principalement, pour la syntaxe, de l'analyse en constituants immédiats (ACI) et des analyses transformationnelles défendues par Chomsky dès ses premiers travaux (Chomsky, 1957).

Riegel et al. (2009, p. 260) adoptent ainsi le point de vue de l'ACI dans leur définition des compléments non essentiels (pour eux « circonstanciels ») :

Troisième constituant majeur de la phrase de base, le complément circonstanciel (ou circonstant) se distingue des deux autres constituants immédiats de la phrase, le GN sujet et le GV, par trois propriétés formelles : il est effaçable, donc facultatif (1), se démultiplie librement (2) et est mobile dans les limites de la phrase entière (3) :

(1) Cette année, l'été a été pluvieux $\rightarrow$ (1a) L'été a été pluvieux.

(1) Cette année, l'été a été pluvieux $\rightarrow(1 b)$ * Cette année a été pluvieux / (1c) * Cette année l'été

(2) Cette année, en Alsace, contrairement aux prévisions de la météo, l'été a été pluvieux, au grand dam des vignerons.

(3a) Au milieu du romantisme, Baudelaire fait songer à quelque classique.

(3b) Baudelaire, au milieu du romantisme, fait songer à quelque classique.

(3c) Baudelaire fait songer à quelque classique, au milieu du romantisme.

(3d) Baudelaire fait songer, au milieu du romantisme, à quelque classique.

20 Ce type de définition domine actuellement dans l'enseignement. On le trouve dans les manuels de linguistique, par exemple chez Monneret (1999). Ce dernier propose ainsi des exercices consistant à isoler dans des phrases les deux ou trois constituants immédiats de la phrase. Exemple (p. 201) : étant donné la phrase :

De nouvelles contraintes apparaissent sur les marchés financiers.

21 l'enjeu est de déterminer si sur les marchés financiers est "complément du verbe » (= complément essentiel) ou " complément circonstanciel » (= non essentiel). Le critère de la « démultiplication » (i.e. le fait qu'on puisse avoir plusieurs compléments circonstanciels) n'est évidemment pas pertinent pour déterminer le statut d'un complément particulier : ajouter par exemple cette année en tête de phrase ne dirait rien sur la fonction de sur les marchés financiers. Seuls l'effacement et le déplacement sont pertinents et ce sont eux que Monneret utilise dans la réponse qu'il préconise :

Le SP sur les marchés financiers est effaçable (de nouvelles contraintes apparaissent) et déplaçable (sur les marchés financiers, de nouvelles contraintes apparaissent). Il doit par conséquent être analysé comme un complément circonstanciel de P. (p. 201).

On notera que la locution par conséquent indique bien que dans l'esprit de ces linguistes, on a la règle :

complément déplaçable et supprimable $\rightarrow$ complément circonstanciel $(=$ non essentiel) 
en linguistique, dont beaucoup visent le professorat des écoles, sont invités à apprendre et utiliser cette règle. Les écoliers aussi, car elle a pris place dans les programmes scolaires. Pellat (2009) indique que le programme officiel 2007 pour le cycle 3 contient la problématique de la « distinction entre complément du verbe, essentiel, et complément de phrase, susceptible d'être supprimé ou déplacé » (p. 57). Dans un manuel destiné au CM2, Hervé et Picot (2010, p. 31, 35, 41, etc.) proposent aux enfants d'« entourer le groupe déplaçable et supprimable s'il y en a un ». C'est moi qui souligne : on voit bien quelles sont les propriétés distinctives du groupe analysé. On demandera ensuite aux enfants de "préciser quelle information il apporte à la phrase ", mais cette deuxième étape n'a pas valeur de définition. Pour tous ces auteurs, c'est le critère formel qui prime, la question du sens vient après.

$\mathrm{Au}$ chapitre des critères syntaxiques qui permettent de distinguer les compléments essentiels et non essentiels, signalons pour être complet le test de dislocation avec et cela (ou avec simplement une coordination, cf. Rémi-Giraud, 1998, p. 81). Si on peut détacher le complément avec et cela, alors il est non essentiel (ex. De nouvelles contraintes apparaissent, et cela sur les marchés financiers), si on ne peut pas, il est essentiel (voir Pellat, 2009 , p. 110 ; Goosse, 1986, § 266).

\subsubsection{Définition des compléments essentiels par recours au sens}

La linguistique qui a influencé la nouvelle grammaire étant une linguistique qui privilégie l'observation de la forme, le point de vue développé dans la section précédente est clairement celui qui domine. En général, cependant, on associe à la définition syntaxique du classement des compléments une définition qui a recours au sens des verbes.

Pour aborder cet angle sémantique de la définition des compléments essentiels, nous partirons des travaux d'Igor Mel'čuk sur son Dictionnaire explicatif et combinatoire (DEC). Pour la discussion qui nous occupe, ces travaux présentent l'intérêt d'être plus développés que ce qu'on trouve dans les grammaires et de sortir du cadre étroit des types de compléments pour intégrer toute la problématique du système lexical. On verra par la suite comment les nouvelles grammaires s'inscrivent dans cette analyse.

Dans le dictionnaire préconisé par Mel'čuk, ce ne sont pas des lexies seules qui sont définies, mais des lexies incluses dans leur forme propositionnelle. La forme propositionnelle d'une lexie est une expression formée de la lexie munie de variables qui renvoient aux « actants sémantiques » de la lexie. Par exemple, on ne définit pas donner (lexie seule), mais $X$ donne $Y$ à $Z$ (lexie dans sa forme propositionnelle); pour vendre, on définit $X$ vend $Y \grave{a} Z$ pour la somme $W$, pour louer, on définit $X$ loue $Y \grave{a} Z$ pour la somme $W$ pendant la période $T$ (voir Mel'čuk et al., 1995, p. 75-79).

On voit que pour les verbes, les variables de la forme propositionnelle correspondent au sujet et à ce qu'on peut voir en première approximation comme les compléments essentiels du verbe. Mel'čuk et al. (1995) précisent en effet :

Soulignons que la présentation de tous les actants sémantiques est indispensable pour préserver le sens voulu dans la définition. Ainsi, si dans la définition de LOUER, on supprime l'actant sémantique $\mathrm{T}$ (= la période de location), le résultat n'est plus 'louer' : c'est 'vendre'; si, dans la définition de VENDRE, on supprime l'actant sémantique W (= la somme d'argent), le résultat n'est plus 'vendre' : c'est 'donner' ; etc. (p. 77) 

et "au sens étroit». Le sens large est celui qui fait du test un moyen d'identifier un complément non essentiel ; c'est le sens commun, qu'on retrouve par exemple dans les exercices de (Monneret, 1999) ou (Hervé et Picot, 2010). Le sens étroit peut se déduire de certains propos de grammairiens qui, s'ils sont pertinents, suggèrent que le test n'est opératoire que pour distinguer un complément de phrase du sujet ou du groupe verbal.

\subsubsection{Au sens large}

Pour fixer l'état de la situation, récapitulons les points vus dans la section 1. Dans le § 1.2.2, à partir des travaux de Mel'čuk, on a caractérisé les compléments essentiels par recours au sens. On a convenu d'appeler ces compléments essentiels au sens. Dans le § 1.2.1, on a exposé les critères syntaxiques mis en avant par les syntacticiens et grammairiens 
modernes, au premier rang desquels le caractère obligatoire ou non. Pour bien distinguer le sens et la forme, convenons d'appeler obligatoire un complément qui doit nécessairement figurer dans une expression avec une lexie $\mathrm{L}$.

On a alors une double opposition essentiel / non essentiel (au sens) et obligatoire / non obligatoire (dans l'expression). En croisant ces deux oppositions, on peut envisager quatre classes théoriques, dont une, la classe des éléments obligatoires et non essentiels au sens, parait vide, ou peut-être simplement peuplée de pronoms qui ne renvoient à rien, comme le pronom se dans s'emparer. Le tableau de la figure 3 illustre ces quatre classes par des exemples. Les parenthèses dans les exemples indiquent que le complément peut être supprimé. Dans la discussion qui suit, on ignorera le cas marginal et douteux des compléments obligatoires et non essentiels au sens (case grisée).

\begin{tabular}{|l|l|l|}
\hline & essentiel au sens & non essentiel au sens \\
\hline obligatoire & $\begin{array}{l}\text { Le bâtiment comporte des parties en } \\
\text { bois. } \\
\text { Il va à Paris. }\end{array}$ & $\begin{array}{l}\text { Deux gardes s'emparèrent du mercier. } \\
\text { Il s'en va. }\end{array}$ \\
\hline $\begin{array}{l}\text { non } \\
\text { obligatoire }\end{array}$ & $\begin{array}{l}\text { Le conseil accepte de (lui) vendre (une } \\
\left.\text { bande de terrain), (au prix de 25 F le } \mathbf{m}^{2}\right) .\end{array}$ & $\begin{array}{l}\text { J'ai visité le pays (en moto). } \\
\text { enfants ont visité (hier), (pour } \\
\text { commencer), la maison d'Eugène } \\
\text { Corbin. }\end{array}$ \\
\hline
\end{tabular}

Figure . Croisement des caractères essentiel au sens et obligatoire

Le problème que pose ce tableau pour la définition des compléments essentiels et non essentiels est qu'il contient non pas deux, mais trois cases pertinentes. S'il n'y avait que les cases obligatoire / essentiel au sens et non obligatoire / non essentiel au sens, tout irait bien, mais la case non obligatoire / essentiel au sens montre que les définitions syntaxiques et sémantiques ne se recoupent pas. Cela, on le sait, je n'énonce rien de nouveau ici, mais on s'en accommode. Il suffit de faire remarquer que les compléments essentiels au sens ne sont pas obligatoires, en laissant penser que les conditions de la non-obligation sont bien cernées :

Un complément essentiel peut le plus souvent être supprimé. [...] L'effacement toujours possible d'un complément essentiel relève d'un type de construction traditionnellement appelé emploi absolu. (Pellat, 2009, p. 110)

ou de signaler l'incertitude de la frontière entre essentiel et non essentiel :

La frontière entre compléments essentiels (nécessaires) et compléments circonstanciels (facultatifs) est parfois ténue, voire discutable. Néanmoins, la Terminologie grammaticale de 1997 invite à distinguer ces deux types de compléments. Cette terminologie officielle apporte une bonne clarification (sic) sur le classement des compléments. (Pellat, 2009, p. 1019)

Il faut admettre le caractère relatif de l'opposition entre essentiel et accessoire. [...] Il existe en fait dans le degré de liaison entre le verbe et un complément un continuum. [...] On ne peut être sûr que [la frontière entre essentiel et accessoire] corresponde toujours à des fonctionnements distincts ou à de véritables enjeux interprétatifs » (Le Goffic, 1993, § 43). 
(2009, p. 109-110) propose trois procédures de reconnaissance pour distinguer les compléments essentiels et les compléments non essentiels. Pour un complément qui désignerait l'acheteur dans une phrase avec vendre, ces trois procédures donnent chacune un résultat différent :

- l'observation de la construction du verbe mène à la conclusion que ce complément est essentiel ;

- l'insertion possible de et cela mène à la conclusion que ce complément est non essentiel ${ }^{7}$;

- l'effacement possible devrait mener à la conclusion que ce complément est non essentiel, mais comme «un complément essentiel peut le plus souvent être supprimé ", l'effacement est non conclusif ${ }^{8}$.

\section{d'effacement est inopérant pour caractériser une distinction sémantique claire et} pertinente ${ }^{11}$.

\subsubsection{Au sens étroit}

La formulation des définitions syntaxiques du « complément de phrase » dans certaines grammaires suggère que le test d'effacement peut ou doit être compris dans un sens plus étroit, qui limite sa portée à un certain contexte. Ainsi, la définition de Riegel et al. ([1994] 2009) citée plus haut, suggère que le caractère effaçable doit se comprendre relativement au sujet et au groupe verbal. Pour illustrer le fait que le complément « circonstanciel » (= non essentiel) est « effaçable, donc facultatif », on montre ces exemples :

(1) Cette année, l'été a été pluvieux $\rightarrow$ (1a) L'été a été pluvieux.

(1) Cette année, l'été a été pluvieux $\rightarrow(1 \mathrm{~b}) *$ Cette année a été pluvieux / (1c) *

Cette année l'été 
1a, 1b, 1c: on teste l'effacement de chacun des trois constituants. Riegel et al. disent: «Troisième constituant majeur de la phrase de base, le complément circonstanciel (ou circonstant) se distingue des deux autres constituants immédiats de la phrase, le GN sujet et le GV, par trois propriétés formelles ». Autrement dit (possiblement) : «Etant donnés les trois constituants d'une phrase, celui qui est effaçable est le circonstanciel». Dans cette interprétation, le test d'effacement ne permet pas d'identifier un constituant, mais seulement de le caractériser; le constituant doit être identifié par ailleurs comme un constituant de la phrase.

L'exemple suivant montrera l'inaptitude du test d'effacement à identifier un groupe comme complément de phrase. Je reproduis ici mot pour mot un raisonnement de Chartrand et al. (1999, p. 66) en changeant simplement la phrase sur lequel il s'applique :

$\mathrm{Y}$ a-t-il un groupe dont la présence est facultative dans cette $\mathrm{P}$ ?

L'équipe a remporté le championnat d'Europe.

L'équipe a remporté le championnat.

Le GP d'Europe est un groupe facultatif. C'est le seul groupe qui peut être effacé sans rendre la phrase asyntaxique. Ce groupe facultatif a la fonction de complément de P. ${ }^{12}$

La phrase originale de Chartrand et al. est L'équipe a remporté le championnat cette année. Elle est présentée avec un découpage de la phrase en trois groupes, matérialisé par des espaces, si bien que la question initiale est à proprement parler « $\mathrm{Y}$ a-t-il un groupe dans les trois groupes identifiés dont la présence est facultative dans cette P?» et que l'observation est : « [Le groupe Cette année] est le seul groupe des trois groupes identifiés qui peut être effacé sans rendre la phrase asyntaxique. »

Si le test d'effacement s'applique pour analyser des groupes identifiés par ailleurs, comment fait-on pour identifier les groupes? La question mérite d'être posée car on utilise peut-être une ou des informations qui rendraient inutiles les tests d'effacement et de déplacement.

\subsection{Critique du test de déplacement}

Le test de déplacement a été critiqué en particulier parce qu'il s'accommode mal des changements de sens que la position du syntagme déplacé induit (cf. Rémi-Giraud, 1998, p. 97-99). Pour Cervoni (1990), ce test « est certainement le plus mauvais de la série » et « il est aberrant de considérer que le complément, après déplacement, est resté ce qu'il était ». Pour Le Goffic (1993), « le critère de la mobilité est clairement inadéquat » (\$ 43) et l'interprétation d'un complément non essentiel est en partie fonction de sa place (§ 309). Ce type de considération aboutit à définir des sous classes de compléments non essentiels par leur portée, qui peut dépendre de leur place: "circonstants de prédicats » et «circonstants de phrase » pour Le Goffic (1993, § 315), compléments circonstanciels intra-, extra-, supra-, et trans- prédicationnels pour Wilmet (2007, § 185).

La classe des compléments non essentiels est pour certains trop hétérogène pour être pertinente. Ainsi, pour Rémi-Giraud (1998, p. 103-104), les connecteurs et les modalisateurs d'énoncés ou d'énonciation devraient être distingués des autres compléments non essentiels car ils sont toujours à l'extérieur du rhème (voir aussi Le Goffic, 1993, § 314-315). Pour Goosse (1986, § 301), qui range ces termes parmi les "éléments incidents", c'est à propos d'eux qu'il serait "plus légitime de parler de complément de phrase ». 
51 A ces critiques bien connues et dont on se demande pourquoi elles restent sans effets, j'ajouterai ici une autre contribution. Elle s'appuie sur une grammaire formelle syntagmatique qui vise à rende compte de la distinction entre complément essentiel et complément non essentiel dans le cas particulier où celui-ci est un groupe nominal.

52 Prenons comme point de départ la grammaire formelle de la figure 4 (14 règles sur deux colonnes).
(1) $P \rightarrow G N G V$
(2) $\mathrm{P} \rightarrow \mathrm{P}$ GN
(8) DET $\rightarrow$ 'le'
(3) $\mathrm{P} \rightarrow \mathrm{GN}$ ',' $\mathrm{P}$
(9) $\mathrm{DET} \rightarrow$ 'la'
(4) $\mathrm{GV} \rightarrow \mathrm{V}$
(10) $\mathrm{N} \rightarrow$ 'nuit'
(5) $\mathrm{V} \rightarrow \mathrm{V} \mathrm{GN}$
(6) GN $\rightarrow$ DET N
(11) $\mathrm{N} \rightarrow$ 'train'
(7) $\mathrm{GN} \rightarrow \mathrm{NP}$
(12) $\mathrm{NP} \rightarrow$ 'Paul'
(13) $\mathrm{V} \rightarrow$ 'dort'
(14) $\mathrm{V} \rightarrow$ 'attend'

Figure. Grammaire formelle de départ.

53 Ce modèle de grammaire est celui qui a été proposé par (Chomsky, 1957, ch.4). Il constitue une formalisation de l'analyse en constituants immédiats. On utilise ici la syntaxe du logiciel NLTK (Bird et al., 2009, ch. 8); le vocabulaire terminal (celui qui compose les phrases spécifiées) est entre guillemets à droite de la flèche dans les règles 8 à 14 ; l'axiome de la grammaire (le symbole du vocabulaire non terminal d'où partent toutes les dérivations) est le premier symbole de la première règle : $P$. Cette grammaire est une grammaire générative; elle spécifie un ensemble de phrases: l'ensemble des séquences de mots du vocabulaire terminal pouvant être construites par application des règles de réécriture à partir du symbole $\mathrm{P}$.

54 La figure 5 donne trois arbres produits par cette grammaire: deux pour la phrase Paul attend la nuit, un pour la phrase la nuit, Paul attend. Les deux arbres associés à Paul attend la nuit rendent compte de l'ambiguïté de cette phrase: dans le premier la nuit est COD de attend, complément essentiel, dans le second, la nuit est « complément de phrase », non essentiel ${ }^{13}$. 

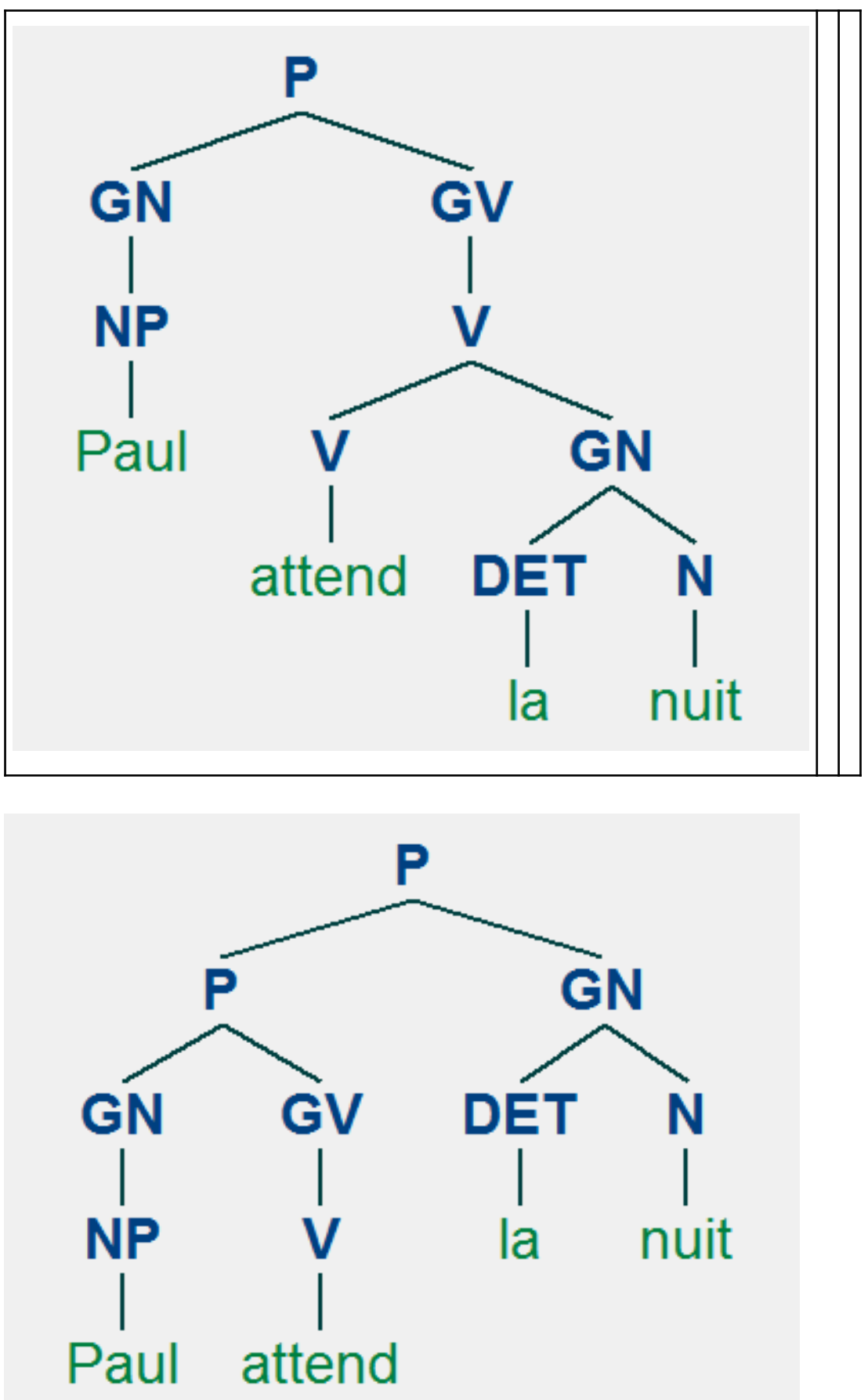


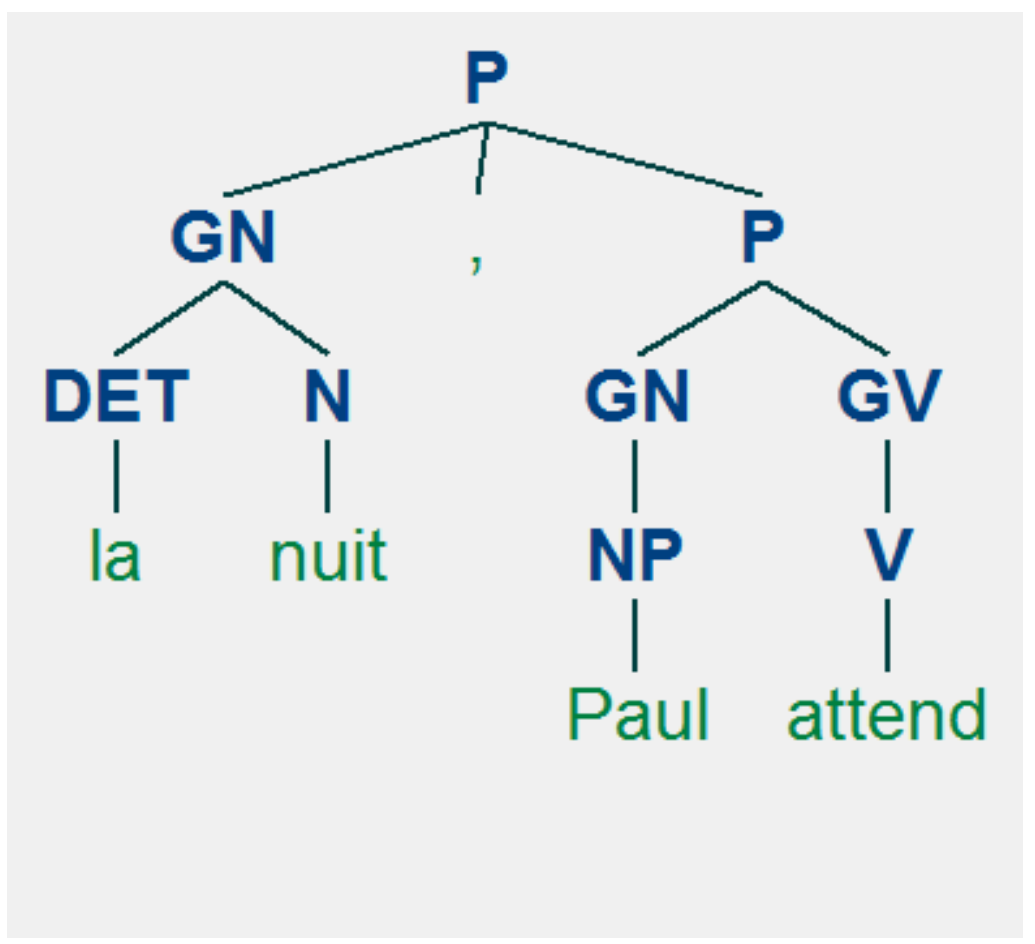

Figure . Arbres associés aux phrases Paul attend la nuit et La nuit, Paul attend par la grammaire de la figure 4. avec ces traits :

Cette grammaire permet de rendre concret le problème des compléments essentiels et non essentiels, soit en fait deux problèmes.

Premier problème : en l'état, la grammaire associe deux arbres à la phrase Paul dort la nuit, comme pour Paul attend la nuit. Or il faut évidemment exclure pour Paul dort la nuit l'arbre où la nuit serait $\mathrm{COD}$ de dort. On peut le faire en recourant à des traits au niveau des règles associées aux verbes, suivant le modèle général des grammaires d'unification (cf. Bird et al., 2009, ch. 9). Les règles touchant les verbes peuvent ainsi, par exemple, être réécrites
(5) $\mathrm{V}[\mathrm{a} 1=0] \rightarrow \mathrm{V}[\mathrm{a} 1=\mathrm{GN}] \mathrm{GN}$
(13) $\mathrm{V}[\mathrm{a} 1=0] \rightarrow$ 'dort'
(14) $\mathrm{V}[\mathrm{a} 1=\mathrm{GN}] \rightarrow$ 'attend' règle 5 pour limiter l'application de cette règle aux seuls verbes qui admettent un $\mathrm{COD}(\mathrm{V}$ [a1=GN] à droite de la flèche). L'arbre où le groupe nominal la nuit serait COD de dort est exclu par ce procédé, mais pas celui où il est COD de attend ${ }^{14}$. dort le train (et deux arbres, un de trop, pour Paul attend le train), car rien n'empêche que le train soit considéré comme un " complément de phrase ».

Comme faire ? Lorsque je pose cette question à mes étudiants de licence SDL $2^{\mathrm{e}}$ année, je peux constater qu'ils ont bien retenu leur leçon du cours de syntaxe suivi par ailleurs, car ils proposent le plus souvent cette réponse : « on regarde si la grammaire admet le train, 
Paul dort, et si elle ne l'admet pas alors la phrase Paul dort le train est jugée agrammaticale ».

Evidemment, une nouvelle question se pose, alors : comment faire pour que la grammaire rejette la phrase le train, Paul dort (car, en l'état, elle l'admet) ? Et là, on voit qu'on ne peut pas faire reposer l'analyse sur les déplacements, car on entre dans un cercle vicieux. En fait, on ne peut pas ne pas faire appel au sens: la phrase Paul dort le train est agrammaticale car le train, de par son sens, est inapte à faire un complément de phrase. Seules quelques lexies dénotant des périodes de temps (ou un lieu) sont aptes à former un GN complément de phrase. Pour rendre compte de la grammaticalité ou agrammaticalité de ces phrases, il faut faire entrer la dimension sémantique du complément.

61 On peut le faire en se donnant un attribut s avec différentes valeurs pour coder le sens, mettons :

$$
\begin{array}{l|l|}
(10) & \mathrm{N}[\mathrm{s}=\mathrm{tps}] \rightarrow \text { 'nuit' } \\
(11) & \mathrm{N}[\mathrm{s}=\mathrm{obj}] \rightarrow \text { 'train' } \\
(12) & \mathrm{NP}[\mathrm{s}=\text { prs }] \rightarrow \text { 'Paul' } \\
\hline
\end{array}
$$

62 et les règles qui créent les GN (6 et 7) et les ajoutent comme compléments de phrase (2 et 3) sont réécrites ainsi :

$$
\begin{array}{|l|l|}
\text { (2) } & \mathrm{P} \rightarrow \mathrm{P} \text { GN[s=tps] } \\
\text { (3) } & \mathrm{P} \rightarrow \mathrm{GN}[\mathrm{s}=\mathrm{tps}] \text { ',' } \mathrm{P} \\
\text { (6) } & \mathrm{GN}[\mathrm{s}=? \mathrm{x}] \rightarrow \mathrm{DET} \mathrm{N}[\mathrm{s}=\text { ?x] } \\
\text { (7) } & \mathrm{GN}[\mathrm{s}=? \mathrm{x}] \rightarrow \mathrm{NP}[\mathrm{s}=? \mathrm{x}]
\end{array}
$$

On exige que le GN complément de phrase ait la valeur tps pour l'attribut $\mathrm{s}$ et les $\mathrm{GN}$ tiennent la valeur de leur attribut $s$ du nom commun ou nom propre utilisé dans la règle 6 ou 7 (?x est une variable qui permet d'assigner à l'attribut $\mathrm{s}$ du GN la valeur de $\mathrm{s} d u \mathrm{~N}$ ou NP ${ }^{15}$.

Evidemment, si on veut traiter les groupes prépositionnels (GP) et groupes adverbiaux (GAdv) compléments, qu'ils soient essentiels ou non, les choses vont se complexifier, mais le raisonnement tenu ici avec un complément d'objet ou complément de phrase de forme GN s'applique aussi pour des compléments de forme GP ou GAdv ${ }^{16}$.

Le principe de ce raisonnement peut se résumer ainsi : on n'interprète pas une phrase en la comparant avec une autre (en testant des manipulations d'effacement ou de déplacement, ou en faisant des transformations), mais en prenant en compte les schémas argumentaux des unités lexicales et l'apport du sens de chaque complément à l'interprétation globale, en général dès qu'on lit ou entend ce complément.

Pour reprendre l'exemple de Monneret (1999) cité plus haut, ce n'est pas parce que sur les marchés financiers peut se placer en tête de phrase qu'il est un " complément de phrase ", c'est parce qu'il dénote le lieu où les contraintes apparaissent. Monneret lui-même reconnait d'ailleurs la primauté du sens : après avoir entrainé ses lecteurs à identifier (et non seulement distinguer les uns des autres) les constituants immédiats de soixante-dix 
phrases grâce aux seuls tests d'effacement et de déplacement, il propose d'étudier une dizaine de phrases ambiguës, où des paraphrases s'ajoutent aux tests dans les explications. La dernière de ces phrases (A la campagne, je préfere la ville) ne peut pas être analysée par les deux tests, parce que à la campagne peut être compris comme essentiel ou non essentiel ${ }^{17}$. Monneret conclut alors (p. 209) : "En l'absence de critères formels efficaces, il n'y a guère d'autre solution que de délaisser le point de vue syntaxique au profit du point de vue sémantique ». En résumé : on présente un critère définitoire, on s'entraine à l'appliquer sur soixante-dix-neuf phrases, et on finit par expliquer que la définition est ailleurs, dans le sens des lexèmes et de leur combinaison.

Je terminerai cette partie par une dernière remarque visant à répondre à une éventuelle objection: la grammaire que j'ai proposée pourrait éventuellement être modifiée pour défendre le concept du déplacement. Au lieu d'avoir les règles 2 et 3 définies ci-dessus, on pourrait n'en conserver qu'une des deux, mettons la (2) :

\begin{tabular}{|l|l|}
\hline$(2)$ & $P \rightarrow$ P GN[s=tps] \\
\hline
\end{tabular}

et remplacer l'autre, la règle (3), par une règle qui transformerait un arbre généré par la règle (2) en un arbre du type que génèrerait la règle (3), sur le modèle du raisonnement que Chomsky (1957) proposait pour les transformations. On voit mal quel serait le gain (1 règle +1 transformation $=2$ règles) et en quoi cette analyse serait cognitivement plus plausible.

\section{Critiques du complément circonstanciel traditionnel}

La nouvelle grammaire s'est imposée non seulement par ses propositions nouvelles, mais aussi par une critique de la tradition. Après avoir présenté une définition traditionnelle du complément circonstanciel, puis évoqué ses origines et son évolution, je relaierai ici les principales critiques qui en sont faites.

\subsection{Une définition traditionnelle de référence : Grevisse}

70 Pour bien voir de quoi il est question, et ne pas tomber dans les définitions erronées du circonstanciel traditionnel que donnent certains grammairiens modernes (voir plus haut, $\S 1.1$ : circonstanciel ne veut pas dire «facultatif»), voici des extraits de la définition des compléments du verbe que donnait Grevisse dans la 11édition du Bon Usage (1980), la dernière avant sa révision par André Goosse (1986).

300. Les compléments du verbe sont: le complément d'objet, le complément circonstanciel et le complément d'agent du verbe passif.

301. Le complément d'objet énonce la personne ou la chose sur laquelle passe l'action du sujet; cette personne ou cette chose est présentée comme supportant l'action, comme étant l'objet de l'action, comme marquant l'aboutissement, l'achèvement du procès : J'éteins LE FEU. Le menteur nuit À SON PROCHAIN. [...]

302. Le complément d'objet direct (on dit aussi simplement objet direct) se rattache ordinairement au verbe directement, sans mot-outil, et exprime, soit l'objet même de l'action, soit le résultat de cette action; avec quelques verbes, il exprime le contenu: Raboter UNE PLANCHE. Enterrer UN TRÉSOR. Graver UNE INSCRIPTION. Enseigner LA GRAMMAIRE. 
Pour identifier le complément d'objet direct, on peut observer qu'il répond à l'une des questions ... qui ?... quoi ? faite après le verbe, et en outre qu'il peut servir de sujet, quand la proposition est tournée par le passif.

Il est préférable, en somme, de consulter le sens, car les deux procédés indiqués ne sont pas toujours efficaces: l'attribut, en effet, répond lui aussi aux questions ... qui ? ... quoi? - et la tournure par le passif ne s'opère pas toujours bien, par exemple avec avoir. [...]

304. Le complément d'objet indirect (on dit aussi simplement objet indirect) est ordinairement rattaché au verbe indirectement, par le moyen d'une préposition; il énonce, en général, la personne ou la chose vers laquelle se dirige l'action : La paresse nuit À LA SANTÉ. Il lègue ses biens À UN HOSPICE. Cet homme obéit À LA NÉCESSITÉ. J'indique le chemin À CE VOYAGEUR. On doute DE SA SINCÉRITÉ.

Pour identifier le complément d'objet indirect, on peut, en consultant le sens, faire après le verbe l'une des questions ... à qui ?... à quoi ? ... de qui ?... de quoi ? etc. [...]

314. Le complément circonstanciel précise l'idée du verbe en marquant la connexion de l'action avec un repère (temps, lieu, etc.) situé autour d'elle dans le monde des phénomènes.

Pour identifier le complément circonstanciel, on fait après le verbe, une des questions ... où ? ... quand ? ... comment ? ... pourquoi ? ... combien ? ... avec quoi ? ... en quoi ? ... par quoi ? ... de combien ? ... par où ? etc.

315. Les circonstances marquées par ce complément sont extrêmement variées. Les principales sont :

[Suit une liste de 33 items : cause, temps (époque, durée), lieu, etc.]

71 Si on compare cette définition avec celle d'une édition plus ancienne du Bon usage (la $6^{\mathrm{e}}$, par exemple, 1955), on peut noter trois différences principales.

La définition générale du complément d’objet (§ 301) était autrefois plus concise : dans la $6^{\mathrm{e}}$ édition (1955), la deuxième proposition était simplement : " cette personne ou cette chose est donc l'objet de l'action ». Pas de référence à l'aboutissement du procès.

Le complément circonstanciel était défini comme « [complétant] l'idée exprimée par le verbe en indiquant les conditions, les circonstances dans lesquelles se trouve le sujet ou s'accomplit l'action du sujet » $(1955, \S 199)$. On voit que, sans doute gêné par le fait de définir circonstanciel par circonstances, Grevisse a substitué à ce dernier terme une glose de son étymologie : circumstare, « être autour ${ }^{18}$.

Enfin, la liste des " principales circonstances » s'est légèrement allongée entre la $6^{\mathrm{e}}$ et la $11^{\mathrm{e}}$ édition : quatre exemples et trois types nouveaux, dont l'un (l'exclusion: Ecrire sans faute(s)) est associé à la privation.

On verra plus loin que ces trois points font écho à des critiques qui condamnent le recours au sens pour les définitions des types de compléments. Outre ces tentatives d'explicitation du sens des types de compléments, l'autre aspect des définitions de Grevisse, qui est resté inchangé entre la $6^{\mathrm{e}}$ et la $11^{\mathrm{e}}$ édition, est l'évocation des questions à utiliser pour identifier ces compléments.

\subsection{Evolution de la définition du circonstanciel}

André Chervel (1979) analyse la genèse du complément circonstanciel en le reliant à trois sources: l'une qui assimile ces compléments aux adverbes, une autre qui leur associe l'«idée accessoire» (p. 8), et une autre qui les associe aux questions diverses auxquelles peuvent répondre les compléments. Selon Chervel, les deux premières sources sont de tradition grammaticale, la troisième est de tradition rhétorique, et c'est cette dernière qui est à l'origine du concept. "A la rhétorique, explique-t-il (1979, p. 12), le 
circonstanciel de notre grammaire doit trois choses: son nom, ses caractéristiques fondamentales et sa procédure d'identification. C'est donc là qu'il trouve sa véritable origine [...]. » Les rhéteurs identifiaient les éléments qu'il convenait de faire entrer dans un discours par une série de questions résumées en un vers latin : Quis, quid, ubi, quibus auxiliis, cur, quomodo, quando ${ }^{19}$. Selon (Chervel, 1979, p. 13), « c'est Beauzée [en 1767] qui fait passer cette grille de la rhétorique à la grammaire et propose pour la première fois de transformer en méthode d'analyse cette procédure ancienne de composition et de création littéraire ».

77 Je résume ici quelques points de l'évolution du concept de complément circonstanciel telle que décrite dans (Chervel, 1977, p. 171-178).

78 1. Au départ (fin XVIII -début XIX ${ }^{\mathrm{e}}$ ), le concept est associé soit avec l'adverbe, soit avec l'idée d'accessoire, et la grammaire scolaire, quand elle utilise le concept, lui donne un rôle tout à fait mineur. L'opposition pertinente est l'opposition entre complément direct et complément indirect. Dans ce contexte, le complément qui suit le verbe dans la phrase Je l'ai vu la veille de son départ est analysé comme indirect, via une ellipse de la préposition: Je l'ai vu (à) la veille de son départ.

2. On cesse d'avoir recours à ce type d'ellipse. Le complément qui suit le verbe dans Je l'ai vu la veille de son départ est alors un complément direct. Pour le distinguer du "véritable complément direct » (le COD), on a recours aux questions : quoi ? quand? « On va appeler désormais "complément circonstanciel" tout complément construit directement ou indirectement, et en relation avec une question [autre que qui ? ou quoi ?].» C'est là le véritable acte de naissance du complément circonstanciel, au milieu du XIX ${ }^{\mathrm{e}}$ siècle.

80 3. Les quatre-cinq questions de base (où, quand, comment, pourquoi, puis combien) n'offrent pas une couverture satisfaisante. "Il n'y avait plus d'interrogatif disponible, explique Chervel (1977, p. 177), alors qu'une foule de nouveaux "circonstanciels" se pressaient à l'horizon des grammairiens. » La liste des types sémantiques s'allonge sans fin.

81 On voit qu'une dérive s'est produite : on est passé des questions à des types sémantiques. La définition de Grevisse (1980) est symptomatique de cette évolution en ce qu'elle met le sens en premier et en ce que Grevisse dit par deux fois qu'il faut «consulter le sens ». Autre symptôme : Rémi-Giraud (1998, p. 66) distingue «trois critères ou sites (points de vue) à partir desquels on peut définir la notion de complément circonstanciel: le site sémantique, le site morphologique et le site syntaxique »; dans les pages qui développent ces trois critères (p. 66-85), on ne trouve aucune référence aux questions, sauf au détour d'une note renvoyant à (Gosselin, 1985). Les questions sont un ancêtre oublié ou négligeable. Puisque seul le sens est finalement retenu comme pertinent, Riegel et al. ([1994] 2009, p. 268) peuvent alors dire qu'« en bonne logique, les grammaires traditionnelles devraient également analyser comme circonstanciel », le complément d'objet « sémantiquement locatif » de Il (re)gagne Paris, comme celui de Il va à Paris.

Pourtant, on peut inverser l'objection de Riegel et al. Si on retient l'usage des questions, les deux compléments sont bien de types différents :

Il regagne quoi ? $\rightarrow$ complément d'objet,

Il va où $? \rightarrow$ complément circonstanciel de lieu.

Le recours aux questions et l'appel à des types sémantiques ne sont pas exactement la même chose et si personne n'a jamais envisagé d'étiqueter le complément de Il (re)gagne Paris comme complément circonstanciel, c'est peut-être bien que les questions sont toujours restées pertinentes pour la définition du concept. 
Recours au sens et questions sont donc les deux pôles de la définition traditionnelle du complément circonstanciel (les définitions, devrait-on dire). On en expose les critiques dans les deux sections suivantes.

\subsection{Critiques du recours au sens}

\subsubsection{Exemples}

Les trois citations qui suivent témoigneront de la défiance des grammairiens modernes à l'égard du recours au sens dans la définition des fonctions en général et dans celle du complément circonstanciel en particulier.

Un verbe peut être accompagné d'un complément dit circonstanciel. On a voulu identifier ce complément en se basant sur le référent: il exprimerait une circonstance, laquelle peut se rapporter au temps, au lieu, à la manière, à la cause, au but, à la mesure, etc. ; on ajoute cet « etc. » parce que personne n'a établi la liste complète des prétendues circonstances. Mais même si cette liste était complète, il resterait à la justifier, à montrer ce qu'il y a de commun à tous ces cas et ce qui les oppose à l'objet, à l'attribut et au complément d'agent. (Buyssens, 1975, p. 34)

Les fonctions doivent être définies au niveau strictement syntaxique, en termes de complémentation, directe ou indirecte. Il faut se méfier du contenu sémantique (largement implicite) des termes habituels de fonction (par exemple « complément d'attribution ») et rechercher le vocabulaire le plus formel et le moins sémantique possible [...]. (Le Goffic, 1993, p. 71)

L'analyse formelle, sémantique et communicative du complément circonstanciel qui précède est aux antipodes des définitions qu'en proposent les grammaires traditionnelles. L'opposition s'explique aisément. Les grammaires traditionnelles définissent en effet la notion de complément circonstanciel sur des bases exclusivement sémantiques et, à l'origine, rhétoriques selon le type de question auquel ils répondent : où ? quand ? comment ? pourquoi ? etc. (A. Chervel, 1979). [... ] Faute de critères formels et, corollairement, d'une définition associative (syntaxique et sémantique) du mode de liaison circonstanciel, la grammaire traditionnelle ne peut valablement distinguer entre compléments du verbe et compléments de la phrase. (Riegel el al., [1994] 2009, p.267)

C'est moi qui souligne les deux occurrences de l'adjectif formel dans ce dernier extrait car c'est en fait seulement sur ce point-là que Riegel et al. se distinguent de la tradition. Seuls les critères formels sont à proprement parler définitoires chez eux, et leur analyse sémantique n'est faite que d'une évocation de quelques types habituels (temps, lieu, cause, etc.) ou nouveaux (rapport à l'énonciation ou à la progression textuelle) et ne se distingue de la tradition que par une incomplétude plus nettement revendiquée :

De toute façon, un tel inventaire, tributaire de la pertinence et de la finesse des distinctions sémantiques opérées, ne sera jamais ni complet ni entièrement satisfaisant. (Riegel et al., [1994] 2009, p. 264)

Deux points saillants ressortent de ces quatre citations : les termes à contenu sémantique sont inadéquats et la liste des types de circonstanciels ne sera jamais complète.

\subsubsection{Inadéquation des termes sémantiques}

Le Goffic (1993) illustre son propos sur le contenu sémantique des termes par le cas particulier du complément d'attribution (emprunter à quelqu'un, donner à quelqu'un), mais il $\mathrm{y}$ a des termes particuliers qui sont assez précis et dont personne n'envisage de se passer - temps, lieu et manière, par exemple. 

Science du langage. Je leur ai soumis une dizaine de phrases en demandant de dire si, oui ou non, le complément en majuscules leur paraissait «marquer la connexion de l'action avec un repère situé autour d'elle dans le monde des phénomènes ». Les résultats sont donnés figure 6 (en pourcentages sur 23 réponses). Que le complément soit un complément d'objet ou un complément circonstanciel, les écarts entre les deux analyses sont assez faibles, proches du hasard, sauf pour Elle habite à Paris.

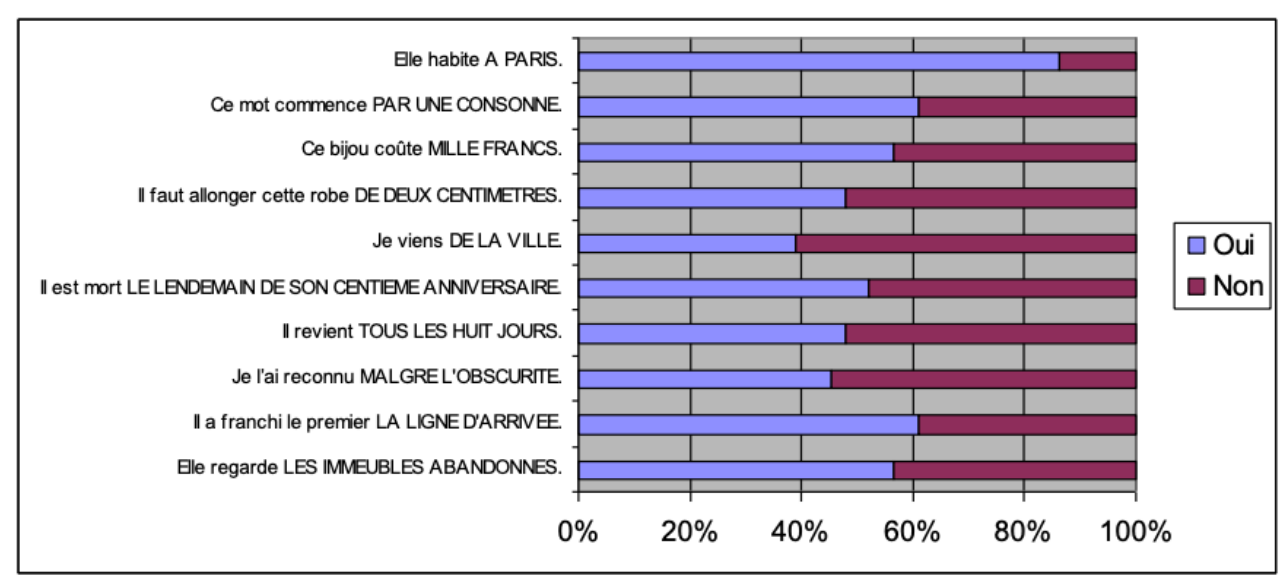

Figure. Analyse de dix compléments comme circonstanciels ou non par 23 étudiants de L1 SDL.

91 Le terme circonstanciel ne peut vraiment pas être pris comme ayant valeur de définition sémantique. Et pourtant, on a fait comme si, et cela a eu une influence considérable dans l'évolution de la grammaire. On a dit plus haut qu'après avoir posé l'usage des quatrecinq questions de base pour identifier les compléments " circonstanciels », " une foule de nouveaux "circonstanciels" se pressaient à l'horizon des grammairiens » (Chervel, 1977, p. 177). Pourquoi ? Parce que les compléments identifiés par les questions s'appelaient "circonstanciels», que ce terme contient l'idée de non essentiel et qu'il fallait donc étendre l'analyse à tous les compléments non essentiels.

92 Toute la problématique de la grammaire moderne sur cette question est de remplacer l'opposition objet / circonstanciel par l'opposition essentiel / non essentiel. Le fait que certains gardent le mot circonstanciel pour les compléments non essentiels ou que Goosse (1986, § 301) se sente tenu de préciser que c'est à eux que « conviendrait l'appellation de complément circonstanciel " est un indice fort que le problème est d'abord une inadéquation terminologique: l'inadéquation du terme circonstanciel à sa définition originelle ${ }^{20}$.

\subsubsection{Incomplétude de la liste des types de circonstanciels}

93 Si on ne peut pas définir le concept de complément circonstanciel par un sens général, peut-être pourrait-on au moins donner la liste de tous les types de compléments particuliers possibles. Là, on objectera que "personne n'a établi la liste complète des prétendues circonstances» (Buyssens, 1975) ou qu'un tel inventaire ne sera jamais complet (Riegel et al., [1994] 2009; voir aussi Le Goffic, 1993, § 310). Quand on voit le dernier ajout de Grevisse (10 édition, 1975) à sa liste des principales circonstances («la 
condition atmosphérique: Voyager par la pluie»), on peut effectivement se demander à quoi rime cette liste. André Goosse a mis fin à l'exercice dans Le bon usage en la réduisant à huit types « utiles, notamment pour l'étude des adverbes et des propositions » $(1986, \S$ 300). Le Goffic (1993, § 279 et $\$ 310)$ propose pour les adverbes « une liste très simple de grands domaines sémantiques » : le lieu, le temps, la quantité et le degré, la manière, et les « énonciatifs » (relations logiques et rapport à l'énonciation), soit cinq classes. A noter que les quatre premières sont définies en relation avec les interrogatifs où, quand, comment et combien.

\subsection{Critiques du recours aux questions}

94 L'origine du recours aux questions constitue l'angle d'attaque principal des critiques qui en sont faites. Il suffit à Riegel et al. de dire «à l'origine rhétorique » pour balayer la procédure comme non pertinente. Dans le même ordre d'idée, Bulea Bronckart et al. (2017) expliquent que dans « l'enseignement grammatical rénové », il s'agit, « au niveau des démarches », "d'abandonner les méthodologies d'inspiration scolastique ». L'adjectif scolastique renvoie au Moyen-Âge, quand la linguistique s'est fondée comme science au Xx e siècle : l'évocation des origines anciennes du recours aux questions a pour effet de placer cette méthode d'analyse hors du champ de la linguistique.

En dehors de cet avis sur les origines qui sonne un peu comme un argument d'autorité, on peut aussi formuler des objections plus précises. Comme elles portent sur des points de détail, on y répondra immédiatement.

Leeman (1998, p. 18) note ces cas de figure :

- une question, plusieurs types sémantique de circonstanciel (ex. pourquoi ? $\rightarrow$ cause ou but) ;

- une question, plusieurs types de complément (ex. comment? peut porter sur un attribut : Ta mère est comment ? - Blonde.) ;

- pas de question pour certains compléments circonstanciels (ex. Il agit par jalousie.).

On peut ranger avec le deuxième cas de figure l'objection que formule Grevisse lui-même $(1980, \S 302)$ sur le fait que l'attribut puisse répondre à la question quoi ? et en tirer la conclusion qu'il faut mettre l'attribut à part parce que les compléments du verbe être ou de ses pairs correspondent à divers interrogatifs (il est quoi ?; il est comment ?; il est où ; il est d'où ?; ils sont combien ?...). Le recours au sens n'est pas indispensable pour cela car la classe des verbes attributifs et les attributs peuvent se définir par référence à la classe de l'adjectif (cf. Buyssens, 1975, p. 33-34 ; Le Goffic, 1993, § 130).

Relève aussi de ce cas, spécifiquement avec la définition de Grevisse (1980), les possibilités ouvertes par le fait que les deux listes données pour l'objet indirect et le circonstanciel se terminent par « etc. ». Donc, pour les prépositions non mentionnées, tout est possible: par exemple, si un complément répond à la question ...contre quoi ?, est-ce un complément d'objet indirect ou un complément circonstanciel? Et même pour les prépositions explicitement mentionnées, on peut être perplexe quand on voit que Discourir D'UNE AFFAIRE et Distinguer le vrai DU FAUX figurent parmi les compléments circonstanciels du $\S 315$, alors qu'ils répondent à la question ...de quoi ? du complément d'objet indirect. Poitevin, à qui Chervel $(1979$, p. 8) attribue la première définition du circonstanciel, en 1844, avait une solution sur ce point: le complément circonstanciel «répond à toute question autre que celle dont on se sert pour le complément direct et le complément 
indirect " ${ }^{21}$. Si on ajoute " ou à aucune question ", on résout également le troisième cas de figure signalé par Leeman.

A ces quelques problèmes finalement assez mineurs, on peut ajouter un problème d'ordre pratique et qui vient du fait que les questions ont l'apparence d'un appel au référent. Soit le texte suivant:

Suite à la demande de Jean-François Legros, la commission des terrains s'est rendue sur place. Après avoir fait part de ses conclusions, le conseil accepte de lui vendre une bande de terrain à proximité de sa résidence, au prix de $25 \mathrm{~F} \mathrm{HT} \mathrm{le} \mathrm{m}^{2}$.

Si on pose sur ce texte la question A qui le conseil accepte-t-il de vendre une bande de terrain?, la réponse sera "à Jean-François Legros" et un observateur non avisé risquera de considérer que l'expression Jean-François Legros est complément d'objet indirect du verbe vendre (alors qu'elle est complément du nom demande). C'est évidemment lui qui est complément de vendre, pronom coréférent avec Jean-François Legros. Il y a ici à prendre en compte les notions de proposition et de pronom et le fait que la réponse à la question n'est valable que dans la mesure où elle est médiée par les expressions du texte. La problématique est sans conteste intéressante et ne justifie pas une mise au rebut de la procédure des questions.

\section{Pour un nouveau classement des compléments du verbe}

Cet article s'intitule Défense du complément circonstanciel parce que je pense que le concept, pourvu qu'on le comprenne comme il a été défini à l'origine, c'est-à-dire par le recours aux questions, est bien plus intéressant que l'opposition fondée sur le caractère effaçable et déplaçable des compléments que défend la nouvelle grammaire ${ }^{22}$. En revanche, on l'a $\mathrm{vu}$, le terme circonstanciel est fondamentalement inadéquat pour l'ensemble de données qu'il était censé couvrir (cf. § 3.3.2). Dans un tel cas, on a deux options : redécouper les données pour qu'elles correspondent aux termes - c'est ce que font les nouveaux grammairiens qui gardent le terme circonstanciel - ou changer les termes pour qu'ils correspondent mieux aux données. Je suivrai cette deuxième option ci-dessous dans la section 4.4 en proposant une "définition restaurée des compléments du verbe». Auparavant, je défendrai le complément circonstanciel traditionnel sur trois points : il ne s’oppose pas à la distinction essentiel / non essentiel (\$ 4.1), les types sémantiques sont nécessaires (au sens fort du terme) ( $\$ 4.2$ ) et le recours aux questions exprime une analyse distributionnelle légitime pour la linguistique moderne (\$ 4.3).

\subsection{Possibilité d'un classement à quatre termes}

102 A la procédure traditionnelle de classement des compléments par recours à des questions, la nouvelle grammaire a substitué des tests d'effacement et déplacement. La première erreur commise par certains promoteurs de la nouvelle grammaire est d'avoir présenté les deux approches comme exclusives l'une de l'autre : puisque le complément de aller est aussi essentiel qu'un complément d'objet, disent-ils en substance, il ne faut pas le distinguer d'un complément d'objet ${ }^{23}$.

103 Pour examiner cette erreur, revenons à la grammaire formelle présentée au § 2.2. Il nous avait fallu ajouter des traits sur les verbes pour que tous les verbes n'admettent pas un 
COD (règle 5) et un trait à interprétation sémantique sur les GN pour que tous les GN ne puissent pas être compléments de phrase (règle 2 ou 3 ) :
(5) $\mathrm{V}[\mathrm{a} 1=0] \rightarrow \mathrm{V}[\mathrm{a} 1=\mathrm{GN}] \mathrm{GN}$
(2) $\mathrm{P} \rightarrow \mathrm{P} \mathrm{GN}[\mathrm{s}=\mathrm{tps}]$

En français, on peut traduire ces apports des traits dans notre grammaire par deux règles :

Règle 1. Un complément essentiel peut être associé au verbe si et seulement si le verbe le demande (= règle 5 ).

Règle 2. Un complément non essentiel peut être ajouté à la phrase si et seulement si il porte en lui une information adéquate (= règle 2 ou 3 ).

On remarquera que dans la première règle, l'information pertinente est sur le complété (le verbe), dans la seconde, l'information pertinente est sur le complément (le GN, dans notre exemple). Par ailleurs, on remarquera aussi que les deux informations exploitées dans les deux règles (sur le complété et sur le complément) ne sont pas exclusives l'une de l'autre. Dans certains cas, elles seront même nécessaires simultanément. Par exemple, le complément de aller, dans son sens de mouvement, doit être un GP ou un GAdv apte à dénoter un lieu (plus précisément : une destination), par exemple : au marché, à Mont-deMarsan, là-bas, vers lui, en enfer, chez un marchand d'étoffes, dans le monde, sur une roche à faible distance, mais pas (ou très difficilement) à soixante-dix ans, ni à Paul Bismuth, ni bien. On peut bien dire que les compléments dans nuire à son prochain et aller à l'école sont tous deux des compléments d'objet indirects, il n'en reste pas moins que les deux verbes n'attendent pas le même paradigme comme complément.

n fait, si on met en parallèle les règles 5 et 2 de la grammaire, on voit que l'opposition essentiel / non essentiel correspond à l'information sur le complété (V demande un complément essentiel, $\mathrm{P}$ admet mais n'exige pas de complément, qui est donc non essentiel) et que l'opposition objet / circonstanciel (au sens traditionnel) correspond à l'information sur le complément. Dans cette optique, le terme objet est peut-être à comprendre non pas comme opposé à sujet, mais comme un terme neutre dans l'ensemble des types sémantiques associés aux compléments ${ }^{24}$. Le $\mathrm{GN}$ de la règle 2 est un circonstanciel car il est marqué, le GN de la règle 5 est un "objet » (concept le plus général qui soit), parce qu'il est non marqué. La différence entre nuire et aller, pour ce qui est des compléments, est que nuire veut la préposition à suivie de n'importe quel GN («objet»: tout est possible, pas de marque) et que aller veut un complément qui corresponde à l'interrogatif où dans le sens « destination » (ensemble restreint, marqué).

La nouvelle grammaire a voulu interpréter le traditionnel circonstanciel comme "non essentiel ». C'est une erreur, parce que c'est mettre au rebut l'idée d'une caractérisation sémantique des compléments, idée qui est à la fois nécessaire (on ne peut pas ne pas faire appel au sens) et compatible avec l'opposition complément essentiel / non essentiel.

Dans sa " petite histoire du circonstanciel », Chervel (1979, p. 12) note que face au choix de mettre en place une opposition à quatre termes : objet / circonstanciel et essentiel / accessoire, la grammaire scolaire a privilégié la première opposition. La nouvelle grammaire scolaire a décidé de privilégier la seconde. Ce n'est pas un progrès, parce que c'est mettre au premier plan des critères de classement formels sans contrepartie 
interprétative (voir plus haut, $\$ 2.1$ ), au détriment de l'analyse des informations indispensables au processus d'interprétation.

\subsection{Nécessité du type sémantique} données.

Je réponds ici aux objections sur le recours au sens. On peut noter que ce qu'on a formulé comme la règle 2 correspond à l'implication

non essentiel $\rightarrow$ sémantiquement marqué

où sémantiquement marqué est une condition nécessaire de non essentiel. Le point clé dans la discussion sur la grammaire était que le trait s=tps (ou un trait analogue pour les GN compléments de lieu) était nécessaire pour que n'importe quel GN ne puisse être pris pour complément circonstanciel. Le même raisonnement doit être tenu pour les adverbes (GAdv) et les groupes prépositionnels (GP). Pour reprendre la formule de (Bonnard, 1982, p.265), le complément circonstanciel est « un complément du verbe [qui porte] en luimême la caractérisation de sa fonction ».

1 A partir de là, il apparait légitime de se demander quelles fonctions sont portées par les GN, GAdv et GP. Puisqu'on a l'implication non essentiel $\rightarrow$ sémantiquement marqué, pourquoi ne pas chercher à caractériser l'inventaire des marques sémantiques? On sait que la tâche est délicate et la nouvelle grammaire rejette cette approche. Le point important, cependant, n'est peut-être pas tant de classer exhaustivement les informations par types, que d'intégrer le fait que les compléments ne sont pas de pures formes. N'importe quel GN, GP ou GAdv ne peut pas être " complément de phrase », le sens qu'il véhicule est une information nécessaire.

12 L'ensemble des types sémantiques associés aux compléments circonstanciels est vaste parce qu'il est conditionné en grande partie par le sens des prépositions, des conjonctions de subordination et des adverbes. A certains égards, le classement des circonstanciels s'apparente à un dictionnaire de ces trois catégories grammaticales. Par exemple, quand Grevisse (1980) identifie ces types de compléments circonstanciels :

L'opposition : Nager contre le courant. Agir contre sa conscience.

La concession : Je te reconnais malgré l'obscurité.

La privation, l'exclusion : Vivre sans pain. Ecrire sans faute(s).

ne fait-il pas que caractériser le sens (ou un des sens) des prépositions contre, malgré et sans? On voit que pour sans, il a besoin de deux termes pour distinguer les variations de sens induites par le nom complément de la préposition. Si on ajoute à ce type de problème le fait que des expressions se figent en locutions conjonctives ou prépositionnelles, on comprend que l'inventaire soit complexe. Nécessairement, on arrivera à des cas où l'identification d'un type englobant deviendra un peu vaine; par exemple, cela vaut-il la peine de se demander quel est le type du complément circonstanciel au grand dam des vignerons?

Cela étant, il n'y a aucune raison pour que les linguistes n'explorent pas ce terrain - si Emile Littré, Pierre Larousse ou Paul Robert s'étaient dit « c'est inutile, parce que de toute façon, un tel inventaire ne sera jamais ni complet ni entièrement satisfaisant », nous n'aurions pas leurs dictionnaires. Par contre, la question se pose de savoir si cette question doit être laissée entièrement aux lexicographes et exclue totalement de la grammaire. Je ferai ci-dessous ( $\$ 4.4)$ une proposition dans le sens d'un partage des 


\subsection{Défense de la commutation} de ne pas voir que quand Beauzée a introduit l'idée d'utiliser les questions de la rhétorique pour analyser les compléments, il faisait de la linguistique distributionnelle. Car il faut comprendre les questions comme des opérations qui font commuter un mot interrogatif avec un syntagme, des opérations sur des expressions. On a déjà donné un aperçu plus haut de la nécessité de prendre en compte l'expression, plutôt que le référent, dans l'usage des questions (cf. la discussion à la fin du § 3.4). Dans son inventaire des sources du circonstanciel, Chervel $(1979$, p. 9) parle de « victoire du critère rhétorique sur la commutation adverbiale ", mais le critère dit « rhétorique » est une commutation souvent adverbiale puisque où, quand, comment et combien sont des adverbes.

117 On peut certes montrer l'ambiguïté de Paul attend la nuit grâce à deux structures différentes, en les justifiant par des tests d'effacement et de déplacement, mais on peut aussi montrer cette ambiguïté en montrant que dans chaque interprétation, le complément ne commute pas avec les mêmes expressions :

Il attend la nuit / le train / cela / quoi?

Il attend la nuit. / après le coucher du soleil / maintenant / quand?

118 La même remarque vaut pour la « transformation passive ». Dans Marchand et al. (1973, p. 40), on oppose Mon père lit le journal et Mon père lit le soir ainsi : « on peut dire Le journal est lu par mon père, et non: *Le soir est lu par mon père.» Certes, mais les interrogatifs quoi et quand donnent un accès plus direct à la distinction ${ }^{25}$ : Mon père lit quoi ?, Mon père lit quand? Pourquoi s'en priver?

119 Tout linguiste sait que la commutation est à la base de l'analyse linguistique moderne. Pourtant, dans la partie de la nouvelle grammaire qui traite de la syntaxe de la phrase, la commutation de base, celle qui place différentes expressions en un même point de l'axe syntagmatique pour identifier un paradigme, est devenue étrangement absente. L'ouvrage de Chartrand et al. (1999) en est un exemple.

La partie dédiée à la grammaire de la phrase de (Chartrand et al., 1999) débute par un chapitre qui donne «les outils d'analyse en grammaire de la phrase ». Parmi ces outils, figurent cinq « manipulations » qu'on peut utiliser pour «mettre en évidence certaines caractéristiques des mots, des groupes, des phrases subordonnées et des $\mathrm{P}$ ». Les trois principales manipulations utilisées sont par ordre de fréquence le remplacement $(39,8 \%)$, l'effacement $(35,4 \%)$ et le déplacement $\left(17,4 \%{ }^{26}\right.$. Le remplacement est l'autre nom de la commutation, l'effacement est la commutation avec le vide ; l'opération de commutation parait donc en bonne place. Pourtant, en dehors de trois remplacements de verbes par être pour caractériser un attribut du sujet et de deux cas très particuliers (ambiguïté de absolument et confusion de quoique et quoi que), tous les remplacements sont des remplacements de pronom ou par un pronom. Dans le tableau synthétique des manipulations pertinentes pour les différentes fonctions syntaxiques (p. 122), le Remplacement est même remplacé par la Pronominalisation. La commutation est réduite à la commutation avec le vide ou avec un pronom, mot qui en lui-même n'a pas de sens.

Corela, $16-2$ | 2018 
121 Pour le « complément de $\mathrm{P}$ », ce choix est extrêmement réducteur : comme il est observé que le « complément de $\mathrm{P}$ ne peut pas être remplacé par un pronom » (p. 105), ne restent pour ce type de constituant que l'effacement et le déplacement. On s'interdit littéralement de pratiquer pour ces constituants des commutations avec des mots grammaticaux autres que les pronoms personnels - les adverbes interrogatifs ou déictiques en particulier, qui offrent des paradigmes plus contrastés que les pronoms personnels. Pourtant, pédagogiquement, rien n'empêcherait de faire travailler des élèves avec ce type de manipulation de façon inductive ${ }^{27}$; l'activité pourrait même être très constructive dans le sens où il s'agirait d'explorer tout ce qui peut se dire, plutôt que ce qui ne se dit pas ${ }^{28}$.

\subsection{Définition restaurée des compléments du verbe}

J'espère avoir montré, à ce point de la discussion, que l'ancienne notion de complément circonstanciel n'était pas sans pertinence ni sans fondement: le sens est une donnée nécessaire des compléments $d u$ verbe, il peut être étudié avec des méthodes distributionnelles.

Pour terminer cet article sur une note constructive, je formulerai une proposition : qu'on reparte de l'idée de Beauzée d'utiliser les questions pour définir des types " sémantiques » de compléments du verbe ${ }^{29}$, mais en prenant garde de contrôler la dérive vers la multiplication de ces types. On évitera la dérive en restant au niveau syntaxique (d'où les guillemets autour du mot sémantiques dans la phrase précédente): les questions sont valables en ce qu'elles mettent en œuvre une commutation d'un syntagme avec un pronom ou adverbe interrogatif. Le paradigme des mots interrogatifs définit ainsi des catégories ${ }^{30}$. Ces mots étant des mots grammaticaux, l'opération semble bien pouvoir être considérée comme relevant de la syntaxe.

\subsubsection{Définitions}

124 1. Les pronoms et adverbes interrogatifs du français définissent cinq catégories principales :

\begin{tabular}{|l|l|}
\hline où & lieu \\
\hline quand & temps \\
\hline combien & quantité \\
\hline comment & manière \\
\hline qui ou quoi & objet \\
\hline
\end{tabular}

Comme Le Goffic (1993) et contrairement à Gosselin (1990), on inclut l'adverbe combien et on met à part l'adverbe pourquoi, qui peut être vu comme composé de la préposition pour + quoi. On considère qui et quoi comme des variantes du même interrogatif, qu'on désignera désormais par quoi. 
2. A l'exception de comment, les interrogatifs peuvent se combiner avec une préposition. Pour où, quand et quoi, ces combinaisons donnent lieu à un sous-classement pertinent.

a. Les possibilités de combinaison de où et quand avec des prépositions sont presque parallèles :

\begin{tabular}{|l|l|l|}
\hline où, entre où et où & situation & quand, entre quand et quand \\
\hline d'où, depuis où & origine & de quand, depuis quand \\
\hline où, jusqu'où, pour où & destination & à quand, jusqu'à quand, pour quand \\
\hline par où & passage & - \\
\hline
\end{tabular}

On remarquera que l'adverbe interrogatif où tout seul est apte à désigner la situation ou la destination, mais qu'il est néanmoins justifié de garder ces deux catégories dans le système. Le latin distinguait les deux par les interrogatifs ubi et quo.

Dans les deux domaines, on peut combiner origine et destination pour définir un intervalle : de où à où, de quand à quand, où les deux syntagmes prépositionnels semblent se comporter comme un seul ${ }^{31}$.

b. Le pronom quoi peut se combiner avec toutes les prépositions et conjonctions de subordination (que je désignerai ensemble par le terme connecteurs). La généralité de quoi et la variété des connecteurs font que le connecteur est susceptible de définir un type sémantique particulier (p. ex. malgré exprime un sens d'opposition), ou des types sémantiques particuliers, par polysémie. La définition de ces types sémantiques relève du dictionnaire des prépositions et conjonctions, mais on note que la conjonction que est vide de sens et que certaines prépositions ont progressivement perdu leur sens précis, en premier lieu à et $d e$ - quand on ne retrouve plus leur sens de «direction vers un point d'aboutissement » et « éloignement d'un point d'origine » encore perceptible avec où et quand, cf. (Riegel et al. [1994] 2009, p. 643).

On peut par conséquent chercher à caractériser les cas où le type général objet reste objet parce que le connecteur n'apporte aucun élément significatif et les cas où ce type se spécialise par l'intermédiaire du connecteur. La tradition a mis en place cette ligne de partage en caractérisant les compléments du verbe qui répondent aux questions à quoi et de quoi comme des compléments d'objet indirects et en mettant dans les circonstanciels les compléments répondant aux questions faites avec une autre préposition + quoi (cf. plus haut, § 3.4, la discussion sur la définition originelle de Poitevin). Cette option n'est peutêtre pas totalement satisfaisante si on considère l'opposition sens vide / sens marqué du connecteur ${ }^{32}$, mais elle est très opératoire. A noter qu'elle est renforcée par un deuxième critère : les compléments d'objet indirects sont « les syntagmes qui se pronominalisent en me, te, se, nous, vous lui, leur » ou "par un en ou un y ne localisant pas le sujet du verbe " (critère de Wilmet, 2007, p. 194).

132 On a utilisé le terme objet en introduction pour désigner la classe des compléments du verbe qui répondent à une question avec quoi, puis on a coupé en deux cette classe avec d'un côté les compléments d'objet (directs et indirects) traditionnels et de l'autre les compléments correspondant à une question en quoi introduite par une préposition autre 
que de ou à ou une conjonction autre que que. La situation pose deux problèmes terminologiques.

Premièrement, si on veut laisser au terme traditionnel de complément d'objet son extension habituelle, on ne peut pas utiliser objet pour la classe englobante associée à l'interrogatif quoi (parce que, par exemple, des compléments correspondant à selon qui ou malgré quoi ne peuvent être désignés comme compléments d'objet sans introduire de confusion inutile). Le problème n'en est pas vraiment un parce qu'en fait, il est peu utile de nommer la classe des compléments qui correspondent à une question en quoi. L'étape en introduction a simplement permis de faire comprendre objet comme terme dans un paradigme où il s'oppose à lieu, temps, etc.

Deuxièmement, il faut nommer le complémentaire des compléments d'objet sur l'ensemble des compléments qui répondent à une question en quoi. Nouveau découpage, nouveau mot; il ne faut surtout pas utiliser circonstanciel qui veut déjà dire trop de choses différentes. Je propose le terme de complément associé, le mot associé étant à comprendre comme indiquant le fait que le complément est introduit par une préposition ou une conjonction qui est (en grande partie) responsable du type sémantique du complément : associé = indirect + une charge sémantique sur le connecteur.

On a donc au final six classes principales : complément d'objet, de quantité, de lieu, de temps, de manière, associé. Ces différentes catégories sont illustrées par des exemples dans la section suivante.

On remarquera que la mise à l'écart de l'interrogatif pourquoi en introduction trouve ici sa justification: les compléments qui correspondent à cette question sont typiquement introduits par une conjonction ou une préposition de sens marqué.

3. Un groupe prépositionnel ou un adverbe complément de verbe qui ne répond à aucune question (p. ex. à mon avis) peut être analysé par convention comme un complément associé.

4. Exceptions : le complément d'agent du verbe passif et les attributs du sujet ou de l'objet constituent des classes à part, pour lesquelles le recours aux questions est non pertinent.

5. Pour certains compléments, on pourra hésiter à les ranger dans l'une ou l'autre classe. Par exemple, le complément de Issu de Jupiter (« extraction » selon Grevisse (1980)) peut être analysé comme un complément d'objet (de qui ?) ou comme un lieu d'origine (d'où ?). Les compléments introduits par avec ou sans peuvent toujours être analysés comme des compléments associés, mais certains peuvent correspondre aussi à la question comment ? : Carreler avec de la brique, Vivre sans pain, Ecrire sans faute, Partir avec un guide (respectivement "matière ", " privation ", « exclusion » et "accompagnement " pour Grevisse (1980)).

\subsubsection{Application}

140 Le tableau qui suit illustre le classement proposé (colonne de gauche) en reprenant les exemples de Grevisse (1980, § 315), à l'exception des cinq exemples à type ambigu qui viennent d'être cités. A droite les types donnés par Grevisse. Quelques remarques suivent le tableau.

lieu

Corela, $16-2$ | 2018 


\begin{tabular}{|c|c|c|}
\hline - situation & Restez chez vous. & situation \\
\hline - origine & $\begin{array}{l}\text { Je viens de la ville. } \\
\text { Suivre de près, de loin le voleur. }\end{array}$ & $\begin{array}{l}\text { point de départ } \\
\text { proximité, éloignement }^{\text {a }}\end{array}$ \\
\hline - destination & Je vais aux champs. & direction \\
\hline - passage & Il s'est introduit par le soupirail. Couper au plus court. & passage \\
\hline temps & & \\
\hline - situation & Nous partirons dans trois jours. & temps (époque) \\
\hline - origine & Sa famille est noble depuis l'an $1400 .{ }^{b}$ & \\
\hline - destination & L'origine de sa noblesse remonte à l'an 1400. & \\
\hline quantité & $\begin{array}{l}\text { Ce bijou coute mille francs. } \\
\text { Ce colis pèse cinq kilos } \\
\text { Il recula de trois pas. } \\
\text { Allonger une robe de deux centimètres. } \\
\text { Travailler toute sa vie. Il resta là trois mois. } \\
\text { Il revient tous les huit jours. }\end{array}$ & $\begin{array}{l}\text { prix } \\
\text { poids } \\
\text { distance } \\
\text { mesure } \\
\text { temps (durée) }{ }^{c} \\
\text { fréquence }\end{array}$ \\
\hline manière & $\begin{array}{l}\text { Il marche à pas pressés. } \\
\text { Il le perça de sa lance. Réussir par la ruse. } \\
\text { Il le prend par la main. }\end{array}$ & $\begin{array}{l}\text { manière } \\
\text { instrument, moyen } \\
\text { partie }\end{array}$ \\
\hline objet & $\begin{array}{l}\text { Discourir d'une affaire. } \\
\text { Distinguer le vrai du faux, l'ami d'avec le flatteur. }\end{array}$ & $\begin{array}{l}\text { propos } \\
\text { séparation }\end{array}$ \\
\hline associé $^{d}$ & $\begin{array}{l}\text { Agir par jalousie. } \\
\text { Il fait cela pour notre édification. } \\
\text { Rendre le bien pour le mal. } \\
\text { Il travaille pour ses enfants. Mettre un terrain en vente. } \\
\text { Egaler quelqu'un en courage. } \\
\text { Nager contre le courant. Agir contre sa conscience. } \\
\text { Je te reconnais malgré l'obscurité. } \\
\text { Il part avec un guide. } \\
\text { Cela m'ennuie à la mort } \\
\text { En cas de besoin, appelez-moi. } \\
\text { Pour un savant, il a fait une étrange erreur. } \\
\text { Se transformer en papillon, changer l'eau en vin. } \\
\text { Voyager par la pluie. }\end{array}$ & $\begin{array}{l}\text { cause } \\
\text { but } \\
\text { échange } \\
\text { destination } \\
\text { point de vue } \\
\text { opposition } \\
\text { concession } \\
\text { accompagnement } \\
\text { conséquence } \\
\text { condition } \\
\text { relativité } \\
\text { changement } \\
\text { condition atmosphérique }\end{array}$ \\
\hline
\end{tabular}

Figure. Exemples de compléments. 
141 a. Les adverbes près et loin sont des adverbes de lieu qui commutent avec où. On voit que c'est chacun des adverbes qui donne le type du complément pour Grevisse.

142 b. J'ajoute cet exemple et le suivant à ceux de Grevisse pour illustrer le parallélisme entre lieu et temps (cf. § 4.4.1, alinéa 2.a). Cela étant, ce sous-classement des compléments de temps parait moins utile que celui des compléments de lieu.

143 c. Ce sont les différentes unités de mesure de quantité qui donnent lieu aux différents types de Grevisse. La durée ne doit pas être classée parmi les compléments de temps, c'est une quantité de temps. La fréquence me semble aussi requérir une unité de mesure ; on dit ordinairement : « tous les combien?».

144 d. Goosse (1986) a écarté le concept de complément circonstanciel. Il utilise celui de complément adverbial, qu'il défini comme « un complément qui est un adverbe ou qui peut être remplacé par un adverbe » (\$299). Il est douteux que tous les compléments de cette ligne du tableau puissent être remplacés par un adverbe.

\subsubsection{Perspectives}

145 Dans une science du réel, la première tâche de l'analyste est de réussir à formuler des observations intersubjectives. Il conviendra donc de tester le système de classement des compléments du verbe proposé ici dans un projet d'annotation de corpus. Le flou régnant dans la typologie traditionnelle des compléments circonstanciels et le rejet d'une caractérisation par le sens des compléments du verbe a fait que dans le projet d'annotation et analyse de corpus que décrit (Guimier, 1993), cette dimension n'a, semblet-il, même pas été envisagée. Est-il possible d'identifier avec précision les compléments de lieu, temps, manière, quantité dans un corpus et de croiser cette information avec le caractère essentiel ou non des compléments que les corpus ciblent en premier lieu ? Si on pouvait obtenir de bons résultats avec une telle typologie simplifiée, ce serait sans doute une information utile.

146 Une autre perspective est bien sûr l'affinage de la typologie proposée. La catégorie des compléments associés est définie en partie morphologiquement (présence d'un connecteur), en partie sémantiquement (le connecteur apporte une partie importante du sens) et en partie par convention (par inclusion de tout ce qui ne répond à aucune question dans les associés).

147 Cette dernière convention peut peut-être être rendue inutile. On a vu (\$ 2.2) que le critère $\mathrm{du}$ « refus de toute forme de rhématisation » (Rémi-Giraud, 1998), qui inclut le fait pour un complément de ne répondre à aucune question, était avancé pour pointer l'hétérogénéité de la classe des compléments non essentiels. La critique vaut aussi pour les compléments associés et inviterait à décomposer cette classe. Par ce critère, seraient exclus des compléments du verbe, et donc des compléments associés, les classes que Goosse (1986) appelle « éléments incidents ", que Wilmet (2007) appelle supra- et transprédicationnels (CP4 et CP5), que Le Goffic (1993, § 279) appelle « adverbes énonciatifs », et qui semblent à peu près les mêmes (il s'agit d'un sous-ensemble des « compléments de phrase " de la nouvelle grammaire). Si la division des adverbes en cinq classes que propose Le Goffic (1993, § 279) est valable, l'opération ne laisserait dans la classe des «associés» que des compléments avec préposition ou conjonction. Le critère de définition morphologique s'en trouverait renforcé. 
Pour ce qui est du critère qui veut que le connecteur apporte une partie importante du sens, il faut reconnaitre qu'il est un peu vague. Il est proposé ici comme le complémentaire de l'idée que de et à seraient des prépositions "vides ", mais la question fait débat (cf. Leeman, 2008, p. 12-13). En tout état de cause, la réflexion sur ce sujet est rendue nécessaire par l'idée qu'un complément non essentiel porte forcément en luimême la caractérisation de sa fonction (non essentiel $\rightarrow$ marqué). Le champ de l'explicitation du sens des prépositions est probablement l'un des domaines les plus complexes de la linguistique, la typologie des compléments que je propose pourrait permettre d'avancer en sortant d'une logique du tout ou rien.

\section{Conclusion}

La grammaire traditionnelle proposait depuis le XIX ${ }^{e}$ siècle et jusqu'au années 1970 un concept de complément circonstanciel que les linguistes modernes s'accordent à reconnaitre comme flou. A la faveur du développement d'une linguistique privilégiant les recherches sur la structure des phrases, ce concept a été écarté des grammaires au profit d'une mise en avant d'une opposition basée sur le caractère essentiel ou non des compléments, se traduisant par des différences structurales.

Bien qu'il soit de nos jours plutôt mal considéré, j'ai essayé de défendre le concept traditionnel de complément circonstanciel - l'idée, pas le nom. J'aboutis à un classement à cinq types "sémantiques" définis par cinq types d'interrogatifs, plus une classe résiduelle. Ce classement a comme mérite de faire revenir dans l'analyse à la fois une dimension interprétative, sans laquelle la langue étudiée n'est pas une langue, et l'opération fondamentale pour l'étude linguistique qu'est la simple commutation. Il présente aussi l'avantage de nous débarrasser du terme complément circonstanciel, mal choisi, inadéquat dès l'origine, tout en clarifiant ce que je crois être le sens premier du concept en grammaire.

151 On peut soupçonner que le terme circonstanciel n'est pas étranger au remplacement de l'opposition objet / circonstanciel par l'opposition essentiel / non essentiel, comme en témoigne l'usage de la première paire de termes pour désigner une opposition du deuxième type chez Riegel et al. ([1994] 2009). Or, on l'a montré, les deux oppositions ne sont nullement exclusives l'une de l'autre. Si on veut les rapprocher (en comprenant essentiel comme " essentiel au sens »), on a cette paire d'implications simples :

objet $\rightarrow$ essentiel

non essentiel $\rightarrow$ non objet ${ }^{33}$

et pas de double implication qui pourrait établir une correspondance absolue. Les nouveaux grammairiens ont donc tort de remplacer l'opposition traditionnelle par l'opposition essentiel / non essentiel.

153 Faut-il choisir l'une ou l'autre opposition ou utiliser les deux conjointement? Je pense avoir montré avec la grammaire formelle du § 2.2 qu'une prise en compte minimale du sens était nécessaire et que les tests d'effacement et de déplacement ne correspondaient à rien dans le processus d'interprétation des phrases. Par ailleurs, j'ai montré (ou plutôt rappelé dans de nouveaux termes, tant le fait est connu) que ces tests échouaient à identifier les compléments qu'on caractérise comme essentiels au sens des verbes. Il semble donc que la combinaison idéale doive être une caractérisation des compléments essentiels par analyse du sens des verbes (cf. § 1.2.2), associée à un typage sémantique des 
compléments du genre présenté ici, c'est-à-dire un typage contrôlé par l'analyse distributionnelle. Dans cette perspective, les différentes places que peuvent occuper les compléments constituent une troisième dimension, qui n'est définitoire ni de l'opposition essentiel / non essentiel, ni d'un sous-type sémantique particulier de complément.

J'ai signalé au début de cet article que dans leur Nouvelle Grammaire du français, Dubois et Lagane (1973) employaient le concept de complément circonstanciel traditionnel sans jamais le définir, comme un acquis. Au $\$ 2.1 .2$, on a vu que certains raisonnements de Riegel et al. ([1994] 2009) et Chartrand et al. (1999) présupposaient que le découpage en constituants était déjà établi. Au § 2.2, on a vu que Monneret (1999) finissait par renvoyer à des critères de sens face à l'inefficacité des tests d'effacement et déplacement. Tous utilisent une information cruciale pour obtenir leurs analyses, mais ils n'en parlent guère, ou secondairement. Il s'agit d'une information à caractère sémantique dont je pense qu'elle n'est pas éloignée de ce que cherchait à cerner la grammaire traditionnelle avec tous ses types de compléments. Du point de vue de la pédagogie, on doit s'interroger sur la pertinence de la mise au premier plan de l'enseignement grammatical de tests qui ne sont au mieux que des outils de méta-analyse, au pire que des «trucs " peu efficaces et favorisant les raisonnements fallacieux, au détriment de l'étude des informations qui entrent effectivement dans le processus d'interprétation.

\section{BIBLIOGRAPHY}

Baillargeon, N. 2007. Petit cours d'autodéfense intellectuelle. Lux.

Beauzée, N. 1767. Grammaire générale, ou Exposition raisonnée des éléments nécessaires du langage : pour servir de fondement à l'étude de toutes les langues.

Bird, S., Klein, E., and Loper, E.. 2009. Natural Language Processing with Python - Analyzing Text with the Natural Language Toolkit. O'Reilly Media and http://www.nltk.org/book/

Blanche-Benvéniste, C., Bilger, M., Rouget, Ch. et Willems, D. 1990. Le français parlé : études grammaticales. Paris, CNRS.

Bonnard, H. 1982. Code du français courant. Magnard.

Bulea Bronckart, E., Marmy Cusin, V. et Panchout-Dubois, M. 2017. « Les exercices grammaticaux dans le cadre de l'enseignement rénové du français : usages, problèmes, perspectives. » In Repères, vol. 56, p. 131-149. https://journals.openedition.org/reperes/1203

Buyssens, E. 1975. Les catégories du français. Bruxelles, Editions de l'Université de Bruxelles.

Cervoni, J. 1990. « Prépositions et compléments prépositionnels ». In Leeman, D. (directrice de publication). Sur les compléments circonstanciels. Langue française, $\mathrm{n}^{\circ} 86$. Larousse.

Chartrand, S.-G., Aubin, D., Blain, R. et Simard, C. 1999. Grammaire pédagogique du français d'aujourd'hui. Montréal, Graficor.

Chervel, A. 1977. Et il fallut apprendre à écrire à tous les petits Français : histoire de la grammaire scolaire. Paris , Payot. 
Chervel, A. 1979. « Rhétorique et grammaire : petite histoire du circonstanciel. » In: Langue française, $n^{\circ} 41$, pp. 5-19;

Chevalier, J.-C. 1968. Histoire de la syntaxe. Naissance de la notion de complément dans la grammaire française (1530-1750). Genève : Droz.

Chomsky, N. 1957. Syntactic Structures. Mouton \& Co.

Dubois, J. et Lagane, R. 1973. La nouvelle grammaire du français. Larousse.

Goosse, A. 1986. Le bon usage. 12e édition. Duculot.

Gosselin, L. 1986. Circonstances et compléments circonstanciels. Doctorat de l'université de Caen.

Gosselin, L. 1990. « Les circonstanciels : de la phrase au texte » In Leeman, D. (dir.). Sur les compléments circonstanciels. Langue française, $\mathrm{n}^{\circ} 86$. Larousse.

Guimier, C. 1993. «L'établissement d'un corpus de circonstants. » In Guimier, C.(dir.). 1001 circonstants. Presses universitaires de Caen.

Grevisse, M. [1936] 1980. Le bon usage. 11e édition. Duculot.

Hervé, L. et Picot, F. 2010. Faire de la grammaire au CM2 cycle 3. Reims : Centre régional de documentation pédagogique de Champagne-Ardenne.

Leeman, D. 1998. Les circonstants en question(s). Editions Kimé.

Leeman, D. 2008. « Prépositions du français : état des lieux », In Langue française, n 157, p. 5-19.

Larousse.

Le Goffic, P. 1993. Grammaire de la phrase française. Paris : Hachette.

Marchand F., Leeman D., Schutte A., Fabre C.. 1973. Comment apprendre la grammaire?

Collection « Apprendre le français » dirigée par J. Dubois et R. Lagane. Larousse.

Mel’čuk I. A., Clas A. et Polguère A. 1995. Introduction à la lexicologie explicative et combinatoire. Duculot, Louvain-la-Neuve.

Monneret, Ph. 1999. Exercices de linguistique. Paris : Presses universitaires de France.

Pellat J.-C., dir. 2009. Quelle grammaire enseigner ? Paris : Hatier.

Rémi-Giraud, S. 1998. « Le complément circonstanciel : problèmes de définition. » In RémiGiraud, S et Roman, A., dir. Autour du circonstant. Presses universitaires de Lyon.

Riegel, M., Pellat, J.-C. et Rioul, R. [1994] 2009. Grammaire méthodique du français. 7e édition. Presses universitaires de France.

Saussure, F. de. [1916] 1995. Cours de linguistique générale. Payot.

Wilmet, M. 2007. Grammaire rénovée du français. De Boeck.

\section{NOTES}

1. Pour des synthèses critiques, voir (Leeman, 1998, ch. 1) et (Rémi-Giraud, 1998).

2. Pellat (2009) utilise aussi la catégorie "complément de phrase » mais elle n'a pas la même extension que chez Chartrand et al. (1999). Pour Pellat (2009), il y a des compléments facultatifs qui dépendent du verbe (les compléments circonstanciels) et des compléments facultatifs qui " portent (au point de vue du sens) sur le reste de la phrase » et sont donc appelés « compléments de phrase» (p. 55). 
3. J'ignore s'ils innovent en cela ou s'ils suivent un usage déjà établi.

4. Le fait que l'ancienne grammaire ne s'intéressait guère à la question du caractère obligatoire ou non de ce complément n'implique en rien qu'elle le considérait facultatif.

5. Comprendre « compléments essentiels » dans la terminologie adoptée au début de cette partie. Pour Riegel et al., les compléments non essentiels ne sont pas compléments du verbe.

6. Je n'ai aucune critique à formuler sur les travaux de Mel'čuk cités ici.

7. Exemple: Le conseil a vendu un terrain, au prix de $25 \mathrm{~F} \mathrm{HT} \mathrm{le} \mathrm{m2,} \mathrm{et} \mathrm{cela} \mathrm{à} \mathrm{un} \mathrm{promoteur}$ immobilier notoirement véreux.

8. Et il n'est pas évident que la non-mention de l'acheteur dans une phrase avec vendre relève de l'emploi absolu.

9. $(p \rightarrow q) \leftrightarrow(\neg q \rightarrow \neg p)$

10. Un parologisme se distingue d'un sophisme en ce qu'il est fait de bonne foi. Baillargeon illustre le parologisme de la négation de l'antécédent avec cet exemple: Si je suis à Londres, je suis en Angleterre. Or je ne suis pas à Londres, donc je ne suis pas en Angleterre.

11. A cette remarque, on peut faire deux objections. 1) Une définition sémantique des compléments essentiels autre que celle que j'ai présentée à partir des travaux de Mel'čuk est peut-être possible. Je n'en ai pas trouvé trace dans les ouvrages consultés. 2) Les tests syntaxiques se suffisent à eux-mêmes et il ne faut pas chercher de correspondance sémantique. Dans ce cas, le jeu parait bien vain. Saussure dirait ([1916], 1995, p. 144) : «L'entité linguistique n'existe que par l'association du signifiant et du signifié; dès qu'on ne retient qu'un de ces éléments, elle s'évanouit; au lieu d'un objet concret, on n'a plus devant soi qu'une pure abstraction ». Par ailleurs, la définition de Mel'čuk parait nécessaire à la description du lexique.

12. Pour faire écho aux critiques de la partie précédente, notez que le raisonnement est aussi mis en défaut avec un « complément de verbe » facultatif.

13. J'adopte dans cette partie le terme "complément de phrase», puisqu'il découle du type d'analyse que matérialise la grammaire de la figure 4.

Détails techniques : dans les grammaires et manuels (p. ex. Riegel et al., [1994] 2009 ; Monneret, 1999), il est plutôt d'usage de considérer que les compléments de phrase (= "circonstanciels » dans les deux ouvrages cités) sont au même niveau que le GN sujet et le GV. La règle qui rendrait compte de cette structure avec un circonstanciel de forme GN pourrait être $\mathrm{P} \rightarrow \mathrm{GN}^{*} \mathrm{GN}$ GV GN*, avec l'étoile indiquant 0 à $\mathrm{n}$ occurrences, mais il n'est pas permis d'utiliser ce type de symbole dans NLTK. Par ailleurs, pour être complet, il faudrait envisager que le circonstanciel puisse se placer entre le sujet et le GV. Ces deux points n'ont aucune incidence sur le fond de la discussion abordée ici.

14. Pour simplifier, on ne traite pas la question du caractère obligatoire ou non du complément. Le trait $a 1=0$ en partie gauche de la règle 5 est là pour empêcher que la règle s'applique récursivement et ajoute des COD à l'infini.

15. Le choix des valeurs prs et obj pour Paul et train, respectivement, n'a pas d'incidence sur la discussion. Il suffit qu'elles soient différentes de tps.

16. Dans (Monneret, 1999) ou (Riegel et al., [1994] 2009), le circonstant est noté toujours GP. C'est un abus de notation. L'étiquette GP ne peut pas à la fois être celle qu'on associe à une séquence préposition + GN dans tous les contextes (c'est son sens fondamental) et celle qu'on associe à un GP, ou un GN, ou un GAdv, dans les contextes où on veut les analyser comme compléments de phrase.

17. Paraphrase $1:$ «Lorsque je suis à la campagne, je préfère la ville. » $\rightarrow$ à la campagne est non essentiel. Paraphrase $2:$ «Je considère la ville comme plus agréable que la campagne.» $\rightarrow$ à la campagne est essentiel.

18. Le changement a été effectué dans la 8e édition (1964).

19. Ce type d'approche est toujours en usage, si l'on en croit l'article QQOQCCP de Wikipédia («Qui ? Quoi ? Où? Quand? Comment? Combien ? Pourquoi ?»). 
20. A cet égard, on peut faire remarquer que les circonstances de la rhétorique incluaient les choses qu'on désigne par le sujet et les compléments d'objet et que les rhéteurs ne peuvent être tenus pour responsables de cette inadéquation.

21. Selon ce critère, les compléments de Discourir d'une affaire et Distinguer le vrai du faux sont des compléments d'objet indirects et non des circonstanciels.

22. Les observations sur les possibilités de placement des compléments sont à garder, mais pour mesurer les effets discursifs induits par les choix de placements possibles, plutôt que pour définir les compléments.

23. «Les compléments de Je vais à Paris et Cela pèse vingt kilos sont liés aussi étroitement au verbe que des compléments d'objet », dit Goosse (1986, § 301).

24. Chevalier (1968, p. 644) évoque un temps (le xviiie siècle) ou le complément d'un verbe transitif pouvait être «le sujet de l'action» (p.ex. avec battre, rompre, tuer) ou «l'objet de l'action» (p.ex. avec voir, entendre, goûter), c'est-à-dire que le terme objet a pu s'inscrire, au moins un temps, dans un paradigme de types sémantiques des compléments.

25. Accès plus direct parce qu'il repose sur l'usage de phrases grammaticales.

26. Calculs effectués sur l'ensemble des manipulations illustrées par une icône dans les chapitres 10 à 27.

27. La découverte des règles par l'induction est un des mots d'ordre de la nouvelle pédagogie de la grammaire. Cf. par exemple (Bulea Bronckart et al., 2017) : "Au niveau des démarches, il s'agissait d'abandonner les méthodologies d'inspiration scolastique pour instaurer des démarches à caractère inductif, accordant une large place à l'activité de l'élève : observer des énoncés, les manipuler, en dégager des régularités, enfin conceptualiser. » Rien n'empêcherait de faire cela dans le cadre de la grammaire traditionnelle, avec de simples commutations.

28. Près d'un quart des manipulations effectuées dans (Chartrand et al., 1999) impliquent une phrase agrammaticale. Certaines semblent parfaitement absurdes, comme cette commutation censée montrer que le complément de $\mathrm{P}$ ne peut être remplacé par un pronom :

Demain, Judith exposera ses meilleures photos.

* $\mathrm{y}$, Judith exposera ses meilleures photos.

29. Au sens très large, qui inclut les « compléments de phrase » de toutes natures.

30. On retrouve cette idée chez Gosselin (1990, p. 39) : «L'analyse par Benveniste des catégories d'Aristote [selon laquelle ces catégories correspondraient, en fait, à des catégories de la langue grecque] peut [...] être considérée positivement et servir de principe à la constitution d'une ontologie revendiquant ses fondements linguistiques : une ontologie sémiologique qui admettrait pour catégories des classes d'expressions partageant un fonctionnement grammatical particulier, irréductible à des propriétés syntaxiques formelles. Concrètement, seront considérées comme appartenant à une même catégorie toutes les réponses possibles à un même type de question. » Voir aussi Le Goffic (1993, § 21 et § 279).

31. Guimier (1993, p. 24) analyse ces deux syntagmes comme un seul groupe.

32. On peut se demander si d'autres prépositions ne peuvent pas être aussi vidées de leur sens que à ou de, comme par exemple les prépositions dans courir après les honneurs ou compter sur son charme (exemples de Riegel et al. [1994] 2009, p. 402). Leeman (2008, p.5) note en parmi les prépositions « vides ».

33. Dans le classement à six termes "sémantiques", non objet = lieu ou temps ou manière ou quantité ou associé. Dans ces deux implications, on peut remplacer objet par non marqué et non objet par marqué. 


\section{ABSTRACTS}

This article confronts two ways of analyzing verb complements in school grammars: the traditional analysis in terms of object and "circumstancial" complements and the analysis as verb (essential) and sentence (non essential) complements of what is called "new grammar". It casts a critical view on the new grammar point of view and defends that of traditional grammar, by showing in particular that, provided one does not distort the original definition, it implements an analysis technique which conforms to standard methods in modern linguistics.

Cet article met en débat deux méthodes d'analyse des compléments du verbe en grammaire scolaire: l'analyse traditionnelle en compléments d'objet et compléments circonstanciels et l'analyse en compléments « du verbe » (essentiels) et « de phrase » (non essentiels) de ce qu'on appelle la «nouvelle grammaire ». Il porte un regard critique sur le point de vue de la nouvelle grammaire et défend celui de la grammaire traditionnelle en montrant notamment que, pour peu qu'on n'en déforme pas la définition initiale, celle-ci met en œuvre une technique d'analyse conforme aux méthodes de la linguistique moderne.

INDEX

Mots-clés: grammaire scolaire, types de compléments, objet, ajout, complément circonstanciel, tests linguistiques

Keywords: school grammar, complement types, object, adjunct, adverbial phrase, linguistic tests

\section{AUTHOR}

\section{FRANÇOIS TROUILLEUX}

Université Clermont Auvergne - LRL (EA 999) 